\title{
A family of generalized quantum entropies: definition and properties
}

\author{
G. M. Bosyk ${ }^{1}$ - S. Zozor ${ }^{2}$ - F. Holik ${ }^{1}$ • \\ M. Portesi ${ }^{1}$ - P. W. Lamberti ${ }^{3}$
}

Received: 1 December 2015 / Accepted: 20 April 2016

(C) Springer Science+Business Media New York 2016

\begin{abstract}
We present a quantum version of the generalized $(h, \phi)$-entropies, introduced by Salicrú et al. for the study of classical probability distributions. We establish their basic properties and show that already known quantum entropies such as von Neumann, and quantum versions of Rényi, Tsallis, and unified entropies, constitute particular classes of the present general quantum Salicrú form. We exhibit that majorization plays a key role in explaining most of their common features. We give a characterization of the quantum $(h, \phi)$-entropies under the action of quantum operations and study their properties for composite systems. We apply these generalized entropies to the problem of detection of quantum entanglement and introduce a discussion on possible generalized conditional entropies as well.
\end{abstract}

Keywords Quantum entropies · Majorization relation · Entanglement detection

\section{Introduction}

During the last decades a vast field of research has emerged, centered on the study of the processing, transmission and storage of quantum information [1-4]. In this field, the

$\bowtie$ G. M. Bosyk

gbosyk@ fisica.unlp.edu.ar

1 Instituto de Física La Plata, UNLP, CONICET, Facultad de Ciencias Exactas, Calle 115 y 49, 1900 La Plata, Argentina

2 Laboratoire Grenoblois d'Image, Parole, Signal et Automatique (GIPSA-Lab, CNRS), 11 rue des Mathématiques, 38402 Saint Martin d'Hères, France

3 Facultad de Matemática, Astronomía y Física, UNC, CONICET, Avenida Medina Allende S/N, Ciudad Universitaria, X5000HUA Córdoba, Argentina 
need of characterizing and determining quantum states stimulated the development of statistical methods that are suitable for their application to the quantum realm [58]. This entails the use of entropic measures particularly adapted for this task. For this reason, quantum versions of many classical entropic measures started to play an increasingly important role, being von Neumann entropy [9] the most famous example, with quantum versions of Rényi [10] and Tsallis [11] entropies as other widely known cases. Many other examples of interest are also available in the literature (see, for instance, [12-14]).

Quantum entropic measures are of use in diverse areas of active research. For example, they find applications as uncertainty measures (as is the case in the study of uncertainty relations [15-20]); in entanglement measuring and detection [2127]; as measures of mutual information [28-31]; and they are of great importance in the theory of quantum coding and quantum information transmission [1,2,3234].

The alluded quantum entropies are nontrivially related, and while they have many properties in common, they also present important differences. In this context, the study of generalizations of entropic measures constitutes an important tool for studying their general properties. In the theory of classical information measures, Salicrú entropies [35] are, up to now, the most generalized extension containing the Shannon [36], Rényi [10] and Tsallis [11] entropies as particular examples and many others as well [37-40]. But a quantum version of Salicrú entropies has not been studied yet in the literature. We accomplish this task by introducing a natural quantum version of the classical expression. Our construction is shown to be of great generality and contains the most important examples (von Neumann, and quantum Rényi and Tsallis entropies, for instance) as particular cases.

We show that several important properties of the classical counterpart are preserved, whereas other new properties are specific of the quantum extension. In our proofs, one of the main properties to be used is the Schur concavity, which plays a key role, in connection with the majorization relation $[25,41]$ for (ordered) eigenvalues of density matrices. Our generalization provides a formal framework which allows to explain why the different quantum entropic measures share many properties, revealing that the majorization relation plays an important role in their formal structure. At the same time, we give concrete clues for the explanation of the origin of their differences. Furthermore, the appropriate quantum extension of generalized entropies can be of use for defining information-theoretic measures suitable for concrete purposes. Given our generalized framework, conditions can then be imposed in order to obtain families of measures satisfying the desired properties.

The paper is organized as follows. In Sect. 2, we give a brief review of (classical) Salicrú entropies, also known as $(h, \phi)$-entropic forms. Our proposal and results are presented in Sect. 3. In Sect. 3.1 we start proposing a quantum version of the $(h, \phi)$ entropies using a natural trace extension of the classical form, followed by the study of its Schur concavity properties in Sect. 3.2. Then, in Sect. 3.3, we study further properties related to quantum operations and the measurement process. In Sect. 3.4 we discuss the properties of quantum entropic measures for the case of composite systems focusing on additivity, sub and superadditivity properties, whereas applications to entanglement detection are given in 3.5. Sect. 4 contains an analysis of informational 
quantities that could be derived from the quantum $(h, \phi)$-entropies. Finally, in Sect. 5, we draw some concluding remarks.

\section{Brief review of classical $(h, \phi)$-entropies}

Inspired by the work of Csiszár [42], Salicrú et al. [35] defined the $(h, \phi)$-entropies:

Definition 1 Let us consider an $N$-dimensional probability vector $p=\left[p_{1} \cdots p_{N}\right]^{t} \in$ $[0,1]^{N}$ with $\sum_{i=1}^{N} p_{i}=1$. The so-called $(h, \phi)$-entropy is defined as

$$
H_{(h, \phi)}(p)=h\left(\sum_{i=1}^{N} \phi\left(p_{i}\right)\right)
$$

where the entropic functionals $h: \mathbb{R} \mapsto \mathbb{R}$ and $\phi:[0,1] \mapsto \mathbb{R}$ are such that either: (i) $h$ is increasing and $\phi$ is concave, or (ii) $h$ is decreasing and $\phi$ is convex. The entropic functional $\phi$ is assumed to be strictly concave/convex, whereas $h$ is taken to be strictly monotone, together with $\phi(0)=0$ and $h(\phi(1))=0$.

We notice that in the original definition [35], the strict concavity/convexity and monotony characters were not imposed. These considerations will allow us to determine the case of equality in some inequalities presented here. The assumption $\phi(0)=0$ is natural in the sense that one can expect the elementary information brought by a zero-probability event to be zero. Also, an appropriate shift in $h$ allows to consider only the case $h(\phi(1))=0$, thus not affecting generality, while giving the vanishing of entropy (i.e., no information) for a situation with certainty.

The $(h, \phi)$-entropies (1) provide a generalization of some well-known entropies such as those given by Shannon [36], Rényi [10], Havrda-Charvát, Daróczy or Tsallis [11,37,38], unified Rathie [39] and Kaniadakis [14], among many others. In Table 1 we list some known entropies and give the entropic functionals $h$ and $\phi$ that lead to these quantities. Notice that the entropies given in the table enter in one (or both) of the special families determined by entropic functionals of the form: $h(x)=x$ and $\phi(x)$ concave [40], or $h(x)=\frac{f(x)}{1-\alpha}$ and $\phi(x)=x^{\alpha}$ [19]. Indeed, the so-called $\phi$-entropy (or trace-form entropy) is defined as

$$
H_{(\mathrm{id}, \phi)}(p)=\sum_{i=1}^{N} \phi\left(p_{i}\right)
$$

where $\phi$ is concave with $\phi(0)=0$, whereas the $(f, \alpha)$-entropy is defined as

$$
F_{(f, \alpha)}(p)=\frac{1}{1-\alpha} f\left(\sum_{i=1}^{N} p_{i}^{\alpha}\right),
$$

where $f$ is increasing with $f(1)=0$, and the entropic parameter $\alpha$ is nonnegative and $\alpha \neq 1$. With the additional assumption that $f$ is differentiable and $f^{\prime}(1)=1$, one recovers the Shannon entropy in the limit $\alpha \rightarrow 1$. 
Table 1 Some well-known particular cases of $(h, \phi)$-entropies

\begin{tabular}{lll}
\hline Name & Entropic functionals & Entropy \\
\hline Shannon & $h(x)=x, \quad \phi(x)=-x \ln x$ & $H(p)=-\sum_{i} p_{i} \ln p_{i}$ \\
Rényi & $h(x)=\frac{\ln (x)}{1-\alpha}, \quad \phi(x)=x^{\alpha}$ & $R_{\alpha}(p)=\frac{1}{1-\alpha} \ln \left(\sum_{i} p_{i}^{\alpha}\right)$ \\
Tsallis & $h(x)=\frac{x-1}{1-\alpha}, \quad \phi(x)=x^{\alpha}$ & $T_{\alpha}(p)=\frac{1}{1-\alpha}\left(\sum_{i} p_{i}^{\alpha}-1\right)$ \\
Unified & $h(x)=\frac{x^{s}-1}{(1-r) s}, \quad \phi(x)=x^{r}$ & $E_{r}^{S}(p)=\frac{1}{(1-r) s}\left[\left(\sum_{i} p_{i}^{r}\right)^{s}-1\right]$ \\
Kaniadakis & $h(x)=x, \quad \phi(x)=\frac{x^{1-\kappa}-x^{1+\kappa}}{2 \kappa}$ & $S_{\kappa}(p)=\sum_{i} \frac{p_{i}^{1-\kappa}-p_{i}^{1+\kappa}}{2 \kappa}$ \\
\hline
\end{tabular}

As recalled in Ref. [19], the $(h, \phi)$-entropies share several properties as functions of the probability vector $p$ :

- $H_{(h, \phi)}(p)$ is invariant under permutation of the components of $p$. Hereafter, we assume that the components of the probability vectors are written in decreasing order.

- $H_{(h, \phi)}\left(\left[p_{1} \cdots p_{N} 0\right]^{t}\right)=H_{(h, \phi)}\left(\left[p_{1} \cdots p_{N}\right]^{t}\right)$ : extending the space by adding zero-probability events does not change the value of the entropy (expansibility property).

- $H_{(h, \phi)}$ decreases when some events (probabilities) are merged, that is, $H_{(h, \phi)}\left(\left[\begin{array}{lllll}p_{1} & p_{2} & p_{3} & \cdots & p_{N}\end{array}\right]^{t}\right) \geq H_{(h, \phi)}\left(\left[p_{1}+p_{2} \quad p_{3} \cdots p_{N}\right]^{t}\right)$. This is a consequence of the Petrović inequality that states that $\phi(a+b) \leq \phi(a)+\phi(b)$ for a concave function $\phi$ vanishing at 0 (and the reverse inequality for convex $\phi$ ) [43, Th. 8.7.1], together with the increasing (resp. decreasing) property of $h$.

Other properties relate to the concept of majorization (see e.g. [44]). Given two probability vectors $p$ and $q$ of length $N$ whose components are set in decreasing order, it is said that $p$ is majorized by $q$ (denoted as $p \prec q$ ), when $\sum_{i=1}^{n} p_{i} \leq \sum_{i=1}^{n} q_{i}$ for all $n=1, \ldots, N-1$ and $\sum_{i=1}^{N} p_{i}=\sum_{i=1}^{N} q_{i}$. By convention, when the vectors do not have the same dimensionality, the shorter one is considered to be completed by zero entries (notice that this will not affect the value of the $(h, \phi)$-entropy due to the expansibility property). The majorization relation allows to demonstrate some properties for the $(h, \phi)$-entropies:

- It is strictly Schur concave: $p \prec q \Rightarrow H_{(h, \phi)}(p) \geq H_{(h, \phi)}(q)$ with equality if and only if $p=q$. This implies that the more concentrated a probability vector is, the less uncertainty it represents (or, in other words, the less information the outcomes will bring). The Schur concavity of $H_{(h, \phi)}$ is consequence of the Karamata theorem [45] that states that if $\phi$ is [strictly] concave (resp. convex), then $p \mapsto \sum_{i} \phi\left(p_{i}\right)$ is [strictly] Schur concave (resp. Schur convex) (see [44, Chap. 3, Prop. C.1] or [46, Th. II.3.1]), together with the [strictly] increasing (resp. decreasing) property of $h$.

- Reciprocally, if $H_{(h, \phi)}(p) \geq H_{(h, \phi)}(q)$ for all pair of entropic functionals $(h, \phi)$, then $p \prec q$. This is an immediate consequence of Karamata theorem [45] (reciprocal part) which states that if for any concave (resp. convex) function $\phi$ one has $\sum_{i=1}^{n} \phi\left(p_{i}\right) \geq \sum_{i=1}^{n} \phi\left(q_{i}\right)$, then $\sum_{i=1}^{n} p_{i} \leq \sum_{i=1}^{n} q_{i}$ for all $n=1, \ldots, N-1$ 
and $\sum_{i=1}^{N} p_{i}=\sum_{i=1}^{N} q_{i}$ (see also [44, A.3-(iv), p. 14 or Ch. 4, Prop. B.1] or [46, Th. II.3.1]).

- It is bounded:

$$
0 \leq H_{(h, \phi)}(p) \leq h\left(\|p\|_{0} \phi\left(\frac{1}{\|p\|_{0}}\right)\right) \leq h\left(N \phi\left(\frac{1}{N}\right)\right),
$$

where $\|p\|_{0}$ stands for the number of nonzero components of the probability vector. The bounds are consequences of the majorization relations valid to any probability vector $p$ (see e.g. [44, p. 9, Eqs. (6)-(8)])

$$
\left[\frac{1}{N} \cdots \frac{1}{N}\right]^{t} \prec\left[\frac{1}{\|p\|_{0}} \cdots \frac{1}{\|p\|_{0}} 0 \cdots \cdots\right]^{t} \prec p \prec\left[\begin{array}{llll}
1 & 0 & \cdots & 0
\end{array}\right]^{t},
$$

together with the Schur concavity of $H_{(h, \phi)}$. From the strict concavity, the bounds are attained if and only if the inequalities in the corresponding majorization relations reduce to equalities.

From the previous discussion, we can see immediately that the $(h, \phi)$-entropies fulfill the first three Shannon-Khinchin axioms [47], which are (in the form given in Ref. [48]) (i) continuity, (ii) maximality (i.e., it is maximum for the uniform probability vector) and (iii) expansibility. The fourth Shannon-Khinchin axiom, the so-called Shannon additivity, is the rule for composite systems that is valid only for the Shannon entropy (notice that there are other axiomatizations of Shannon entropy, e.g., those given by Shannon in [36] or by Fadeev in [49]). A relaxation of Shannon additivity axiom, called composability axiom, has been introduced [48,50]; it establishes that the entropy of a composite system should be a function only of the entropies of the subsystems and a set of parameters. The class of entropies that satisfy these axioms (the first three Shannon-Khinchin axioms and the composability one) is wide [51] but nevertheless can be viewed as a subclass of the $(h, \phi)$-entropies.

It has recently been shown that the $(h, \phi)$-entropies can be of use, for instance, in the study of entropic formulations of the quantum mechanics uncertainty principle [18, 19]. They have also been applied in the entropic formulation of noise-disturbance uncertainty relations [52]. Our aim is to extend the definition of the $(h, \phi)$-entropies for quantum density operators and study their properties and potential applications in entanglement detection.

\section{Quantum $(h, \phi)$-entropies}

\subsection{Definition and link with the classical entropies}

The von Neumann entropy [9] can be viewed as the quantum version of the classical Shannon entropy [36], by replacing the sum operation with a trace. We recall that for an Hermitian operator $A=\sum_{i} a_{i}\left|a_{i}\right\rangle\left\langle a_{i}\right|$, with $\left|a_{i}\right\rangle$ being its orthonormal eigenvectors in $\mathcal{H}^{N}$ and $a_{i}$ being the corresponding eigenvalues, one has $\phi(A)=\sum_{i} \phi\left(a_{i}\right)\left|a_{i}\right\rangle\left\langle a_{i}\right|$, and the trace operation is the sum of the corresponding eigenvalues (i.e., $\operatorname{Tr} \phi(A)=$ 
$\sum_{i} \phi\left(a_{i}\right)$, where $\operatorname{Tr}$ stands for the trace operation). In a similar way to the classical generalized entropies, we propose the following definition:

Definition 2 Let us consider a quantum system described by a density operator $\rho$ acting on an $N$-dimensional Hilbert space $\mathcal{H}^{N}$, which is Hermitian, positive (that is, $\rho \geq 0$ ), and with $\operatorname{Tr} \rho=1$. We define the quantum $(h, \phi)$-entropy as follows

$$
\mathbf{H}_{(h, \phi)}(\rho)=h(\operatorname{Tr} \phi(\rho)),
$$

where the entropic functionals $h: \mathbb{R} \mapsto \mathbb{R}$ and $\phi:[0,1] \mapsto \mathbb{R}$ are such that either: (i) $h$ is strictly increasing and $\phi$ is strictly concave, or (ii) $h$ is strictly decreasing and $\phi$ is strictly convex. In addition, we impose $\phi(0)=0$ and $h(\phi(1))=0$.

The link between Eqs. (1) and (5) is the following. Let us consider the density operator written in diagonal form (spectral decomposition) as $\rho=\sum_{i=1}^{N} \lambda_{i}\left|e_{i}\right\rangle\left\langle e_{i}\right|$, with eigenvalues $\lambda_{i} \geq 0$ satisfying $\sum_{i=1}^{N} \lambda_{i}=1$, and being $\left\{\left|e_{i}\right\rangle\right\}_{i=1}^{N}$ an orthonormal basis. Then, the quantum $(h, \phi)$-entropy can be computed as

$$
\mathbf{H}_{(h, \phi)}(\rho)=H_{(h, \phi)}(\lambda) .
$$

This equation states that the quantum $(h, \phi)$-entropy of a density operator $\rho$, is nothing but the classical $(h, \phi)$-entropy of the probability vector $\lambda$ formed by the eigenvalues of $\rho$. Notice that despite the link between the quantal and the classical entropies defined from a pair of entropic functionals $(h, \phi)$, we keep a different notation for the entropies ( $\mathbf{H}$ and $H$, respectively) in order to distinguish their very different meanings.

The most relevant examples of quantum entropies, which are the von Neumann one [9], quantum versions of the Rényi, Tsallis, unified and Kaniadakis entropies [13, $27,53,54]$, and the quantum entropies proposed in Refs. [12,55], are clearly particular cases of our quantum $(h, \phi)$-entropies (5).

In what follows, we give some general properties of the quantum $(h, \phi)$-entropies (the validity of the properties for the von Neumann entropy is already known, see for example $[3,25,56-58])$. In our derivations, we often exploit the link (6). With that purpose, hereafter we consider, without loss of generality, that the eigenvalues of a density operator $\rho$ are arranged in a (probability) vector $\lambda$, with components written in decreasing order.

\subsection{Schur concavity, concavity and bounds}

One of the main properties of the classical $(h, \phi)$-entropies, namely the Schur concavity, is preserved in the quantum version of these entropies:

Proposition 1 Let $\rho$ and $\rho^{\prime}$ be two density operators, acting on $\mathcal{H}^{N}$ and $\mathcal{H}^{N^{\prime}}$ respectively, and such that $\rho \prec \rho^{\prime}$. Then

$$
\mathbf{H}_{(h, \phi)}(\rho) \geq \mathbf{H}_{(h, \phi)}\left(\rho^{\prime}\right),
$$


with equality if and only if either $\rho^{\prime}=U \rho U^{\dagger}$, or $\rho=U \rho^{\prime} U^{\dagger}$, for any isometric operator $U$ (i.e., $U^{\dagger} U=I$ ), where $U^{\dagger}$ stands for the adjoint of $U$. Reciprocally, if Eq. (7) is satisfied for all pair of entropic functionals, then $\rho \prec \rho^{\prime}$.

Proof Let $\lambda$ and $\lambda^{\prime}$ be the vectors of eigenvalues of $\rho$ and $\rho^{\prime}$, respectively, rearranged in decreasing order and adequately completed with zeros to equate their lengths. By definition, $\rho \prec \rho^{\prime}$ means that $\lambda \prec \lambda^{\prime}$ (see [25, p. 314, Eq. (12.9)]). Thus, the Schur concavity of the quantum $(h, \phi)$-entropy (and the reciprocal property) is inherited from that of the corresponding classical $(h, \phi)$-entropy, due to the link (6). From the strict concavity or convexity of $\phi$ and thus the strict Schur concavity of the classical $(h, \phi)$-entropies, the equality holds in (7) if and only if $\lambda^{\prime}=\lambda$, that is equivalent to have either $\rho^{\prime}=U \rho U^{\dagger}$ (when $N \leq N^{\prime}$ ) or $\rho=U \rho^{\prime} U^{\dagger}\left(\right.$ when $\left.N^{\prime} \leq N\right)$.

As a direct consequence, the quantum $(h, \phi)$-entropy is lower and upper bounded, as in the classical case:

Proposition 2 The quantum $(h, \phi)$-entropy is lower and upper bounded

$$
0 \leq \mathbf{H}_{(h, \phi)}(\rho) \leq h\left(\operatorname{rank} \rho \phi\left(\frac{1}{\operatorname{rank} \rho}\right)\right) \leq h\left(N \phi\left(\frac{1}{N}\right)\right)
$$

where rank stands for the rank of an operator (the number of nonzero eigenvalues). Moreover, the lower bound is achieved only for pure states, whereas the upper bounds are achieved for a density operator of the form $\rho=\frac{1}{M} \sum_{i=1}^{M}\left|e_{i}\right\rangle\left\langle e_{i}\right|$ for some orthonormal ensemble $\left\{\left|e_{i}\right\rangle\right\}_{i=1}^{M}$, with $M=\operatorname{rank} \rho$ in the tightest situation and $M=N$ in the other one (in the latter case, necessarily $\rho=\frac{1}{N} I_{N}$, being $I_{N}$ the identity operator in $\mathcal{H}^{N}$ ).

Proof Let $\lambda$ be the vector formed by the eigenvalues of $\rho$. Clearly rank $\rho=\|\lambda\|_{0}$, so that the bounds are immediately obtained from that of the classical $(h, \phi)$-entropy, due to the link (6). Moreover, in the classical case $H_{(h, \phi)}(\lambda)=0$ if and only if $\lambda=\left[\begin{array}{llll}1 & 0 & \cdots & 0\end{array}\right]^{t}$, that is $\rho=|\Psi\rangle\langle\Psi|$ is a pure state. On the other hand, the upper bounds are attained if and only if $\lambda=[\underbrace{\frac{1}{M} \cdots \frac{1}{M}}_{M} 0 \cdots 0]^{t}$, with $M=\operatorname{rank} \rho$ or $M=N$

The classical $(h, \phi)$-entropies and their quantum versions are generally not concave. We establish here sufficient conditions on the entropic functional $h$ to ensure the concavity property of the quantum $(h, \phi)$-entropies. We notice that, with the same sufficient conditions, the classical counterpart is also concave:

Proposition 3 If the entropic functional $h$ is concave, then the quantum $(h, \phi)$-entropy is concave, that is, for all $0 \leq \omega \leq 1$,

$$
\mathbf{H}_{(h, \phi)}\left(\omega \rho+(1-\omega) \rho^{\prime}\right) \geq \omega \mathbf{H}_{(h, \phi)}(\rho)+(1-\omega) \mathbf{H}_{(h, \phi)}\left(\rho^{\prime}\right) .
$$


Proof Let us first recall the Peierls inequality (see [25, p. 300]): if $\phi$ is a convex function and $\sigma$ is an Hermitian operator acting on $\mathcal{H}^{N}$, then for any arbitrary orthonormal basis $\left\{\left|f_{i}\right\rangle\right\}_{i=1}^{N}$, the following inequality holds

$$
\operatorname{Tr} \phi(\sigma) \geq \sum_{i} \phi\left(\left\langle f_{i}|\sigma| f_{i}\right\rangle\right)
$$

Consider $\sigma=\omega \rho+(1-\omega) \rho^{\prime}=\sum_{i} \lambda_{i}\left|e_{i}\right\rangle\left\langle e_{i}\right|$ written in its diagonal form, $h$ decreasing and $\phi$ convex. Then

$$
\begin{array}{rlr}
\operatorname{Tr} \phi(\sigma) & =\sum_{i} \phi\left(\lambda_{i}\right)=\sum_{i} \phi\left(\left\langle e_{i}|\sigma| e_{i}\right\rangle\right) \\
& =\sum_{i} \phi\left(\left\langle e_{i}\left|\left[\omega \rho+(1-\omega) \rho^{\prime}\right]\right| e_{i}\right\rangle\right) \\
& \leq \omega \sum_{i} \phi\left(\left\langle e_{i}|\rho| e_{i}\right\rangle\right)+(1-\omega) \sum_{i} \phi\left(\left\langle e_{i}\left|\rho^{\prime}\right| e_{i}\right\rangle\right) & {[\phi \text { being convex] }} \\
& \leq \omega \operatorname{Tr} \phi(\rho)+(1-\omega) \operatorname{Tr} \phi\left(\rho^{\prime}\right)^{2} & \text { [due to Peierls inequality]. }
\end{array}
$$

Notice that in the case $\phi$ concave, these two inequalities are reversed. Thus, one finally has

$$
\begin{array}{rlrl}
h(\operatorname{Tr} \phi(\sigma)) & \geq h\left(\omega \operatorname{Tr} \phi(\rho)+(1-\omega) \operatorname{Tr} \phi\left(\rho^{\prime}\right)\right) & & {[h \text { being decreasing }]} \\
& \geq \omega h(\operatorname{Tr} \phi(\rho))+(1-\omega) h\left(\operatorname{Tr} \phi\left(\rho^{\prime}\right)\right) & \text { [assuming } h \text { concave] }
\end{array}
$$

Notice that in the case $h$ increasing, the first inequality holds, and the second inequality holds as well, from concavity of $h$ (with equality valid when $h$ is the identity function). Making use of Definition 2, the proposition is proved in both cases, under the condition that the entropic functional $h$ is concave.

Note also that for the class of $(f, \alpha)$-entropies, the concavity of $h$ is equivalent to that of $\frac{f}{1-\alpha}$. Moreover, for the von Neumann and quantum Tsallis entropies, the conditions of Proposition 3 are satisfied, and it is well known that these entropies have the concavity property. For quantum Rényi entropies, the concavity property holds for $0<\alpha<1$ as consequence of Proposition 3, but for $\alpha>1$ the proposition does not apply (see [25, p. 53] for an analysis of concavity in this range for classical Rényi entropies). For the quantum unified entropies, the concavity property holds in the range of parameters $r<1$ and $s<1$ or $r>1$ and $s>1$ as consequence of Proposition 3, which complements the result of Ref. [13] and improves the result of Ref. [53].

It is interesting to remark that using the concavity property given in Proposition 3, it is possible to define in a natural way, for $h$ concave, a (Jensen-like) quantum $(h, \phi)$ divergence between density operators $\rho$ and $\rho^{\prime}$, as follows:

$$
\mathbf{J}_{(h, \phi)}\left(\rho, \rho^{\prime}\right)=\mathbf{H}_{(h, \phi)}\left(\frac{\rho+\rho^{\prime}}{2}\right)-\frac{1}{2}\left[\mathbf{H}_{(h, \phi)}(\rho)+\mathbf{H}_{(h, \phi)}\left(\rho^{\prime}\right)\right],
$$

which is nonnegative and symmetric in its arguments. This is similar to the construction presented in Ref. [40] for the classical case, and offers an alternative to the quantum 
version of the usual Csiszár divergence $[42,55]$. It can be shown that for pure sates $|\psi\rangle$ and $\left|\psi^{\prime}\right\rangle$ the quantum $(h, \phi)$-divergence (11) takes the form

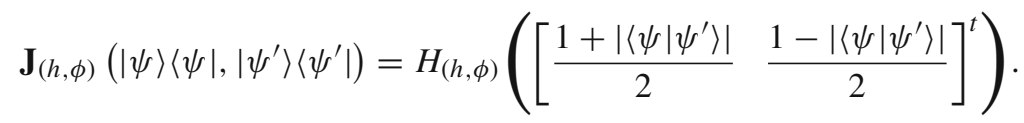

Indeed, the square root of this quantity in the von Neumann case $[h(x)=x, \phi(x)=$ $-x \ln x$ ] provides a metric for pure states [59]. Notice that the right-hand side of Eq. (12) is a binary $(h, \phi)$-entropy. Other basic properties and applications of the quantum $(h, \phi)$-divergence are currently under study [60].

\subsection{Specific properties of the quantum $(h, \phi)$-entropy}

We recall that the quantum entropy of a density operator equals the classical entropy of the probability vector formed by its eigenvalues. In other words, considering a density operator as a mixture of orthonormal pure states, its quantum entropy coincides with the classical entropy of the weights of the pure states. This is not true when the density operator is not decomposed in its diagonal form, but as a convex combination of pure states that do not form an orthonormal basis. The quantum $(h, \phi)$-entropy of an arbitrary statistical mixture of pure states is upper bounded by the classical $(h, \phi)$ entropy of the probability vector formed by the mixture weights:

Proposition 4 Let $\rho=\sum_{i=1}^{M} p_{i}\left|\psi_{i}\right\rangle\left\langle\psi_{i}\right|$ be an arbitrary statistical mixture of pure states $\left|\psi_{i}\right\rangle\left\langle\psi_{i}\right|$, with $p_{i} \geq 0$ and $\sum_{i=1}^{M} p_{i}=1$. Then, the quantum $(h, \phi)$-entropy is upper bounded as

$$
\mathbf{H}_{(h, \phi)}(\rho) \leq H_{(h, \phi)}(p),
$$

where $p=\left[p_{1} \cdots p_{M}\right]^{t}$.

Proof First, we recall the Schrödinger mixture theorem [25, Th. 8.2]: a density operator in its diagonal form $\rho=\sum_{i=1}^{N} \lambda_{i}\left|e_{i}\right\rangle\left\langle e_{i}\right|$ can be written as an arbitrary statistical mixture of pure states $\rho=\sum_{i=1}^{M} p_{i}\left|\psi_{i}\right\rangle\left\langle\psi_{i}\right|$, with $p_{i} \geq 0$ and $\sum_{i=1}^{M} p_{i}=1$, if and only if, there exist a unitary $M \times M$ matrix $U$ such that

$$
\sqrt{p_{i}}\left|\psi_{i}\right\rangle=\sum_{j=1}^{N} U_{i j} \sqrt{\lambda_{j}}\left|e_{j}\right\rangle
$$

As a corollary, one directly has [61]

$$
p=B \lambda
$$


where $B_{i j}=\left|U_{i j}\right|^{2}$ are the elements of the $M \times M$ bistochastic matrix ${ }^{1} B$. From the lemma of Hardy, Littlewood and Pólya [25, Lemma 2.1] or [44, Th. A.4], this is equivalent to the majorization relation $p \prec \lambda$. Therefore, from (6) and the Schur concavity of the classical $(h, \phi)$-entropy, we immediately have $\mathbf{H}_{(h, \phi)}(\rho)=H_{(h, \phi)}(\lambda) \leq$ $H_{(h, \phi)}(p)$.

The previous proposition is a natural generalization of a well-known property of von Neumann entropy. One can also show that a related inequality holds:

Proposition 5 Let $\left\{\left|e_{k}\right\rangle\right\}_{k=1}^{N}$ be an arbitrary orthonormal basis of $\mathcal{H}^{N}$ and, for a given density operator $\rho$ acting on $\mathcal{H}^{N}$, let us denote by $p^{E}(\rho)$ the probability vector with elements $p_{k}^{E}(\rho)=\left\langle e_{k}|\rho| e_{k}\right\rangle$, that is, the diagonal elements of $\rho$ related to that basis. Then

$$
\mathbf{H}_{(h, \phi)}(\rho) \leq H_{(h, \phi)}\left(p^{E}(\rho)\right) .
$$

Proof The decomposing of $\rho$ in the basis $\left\{\left|e_{k}\right\rangle\right\}_{k=1}^{N}$ has the form $\rho=\sum_{k, l=1}^{N} \rho_{k, l}\left|e_{k}\right\rangle\left\langle e_{l}\right|$ where the diagonal terms are $\rho_{k, k}=p_{k}^{E}(\rho)$. The Schur-Horn theorem [25, Th. 12.4] states that the vector $p^{E}(\rho)$ of the diagonal terms of $\rho$ is majorized by the vector $\lambda$ of the eigenvalues of $\rho$. Thus, from the Schur concavity property of the classical $(h, \phi)$-entropy, we have $\mathbf{H}_{(h, \phi)}(\rho)=H_{(h, \phi)}(\lambda) \leq H_{(h, \phi)}\left(p^{E}(\rho)\right)$.

We consider now the effects of transformations. Among them, unitary operators are important since the time evolution of an isolated quantum system is described by a unitary transformation (i.e., implemented via the action of a unitary operator on the state). One may expect that a "good" entropic measure remains unchanged under such a transformation. This property, known to be valid for von Neumann and quantum Rényi entropies [62] among others, is fulfilled for the quantum $(h, \phi)$-entropies, and even in a slightly stronger form, i.e., for isometries. We recall that an operator $U: \mathcal{H}^{N} \mapsto \mathcal{H}^{N^{\prime}}$ is said to be isometric if it is norm preserving. This is equivalent to $U^{\dagger} U=I$. On the other hand, an operator is then said to be unitary if it is both isometric and coisometric, that is, both $U$ and $U^{\dagger}$ are isometric. When $U: \mathcal{H}^{N} \mapsto \mathcal{H}^{N}$ (both Hilbert spaces having the same dimension) is isometric, it is necessarily unitary (see e.g. [63]).

Proposition 6 The quantum $(h, \phi)$-entropy is invariant under any isometric transformation $\rho \mapsto U \rho U^{\dagger}$ where $U$ is an isometric operator:

$$
\mathbf{H}_{(h, \phi)}\left(U \rho U^{\dagger}\right)=\mathbf{H}_{(h, \phi)}(\rho) .
$$

Proof Let us write $\rho$ in its diagonal form, $\rho=\sum_{i=1}^{N} \lambda_{i}\left|e_{i}\right\rangle\left\langle e_{i}\right|$. Clearly, $U \rho U^{\dagger}=$ $\sum_{i=1}^{N} \lambda_{i}\left|f_{i}\right\rangle\left\langle f_{i}\right|$, where $\left|f_{i}\right\rangle=U\left|e_{i}\right\rangle$ with $i=1, \ldots, N$, form an orthonormal basis (due to the fact that $U$ is an isometry). Since $\rho$ and $U \rho U^{\dagger}$ have the same eigenvalues, and thus, using Eq. (6), we conclude that they have the same $(h, \phi)$-entropy.

\footnotetext{
${ }^{1}$ It is assumed that $M \geq N$, otherwise $p$ is completed with zeros; when $M>N$, the remaining $N-M$ terms that do not appear in Eq. (14) are added in order to fulfill the unitary of $U$ and $\lambda$ is to be understood as completed with zeros (for more details, see the proof of the Schrödinger mixture theorem [25, pp. 222-223]).
} 
When dealing with a quantum system, it is of interest to estimate the impact of a quantum operation on it. In particular, one may guess that a measurement can only perturb the state and, thus, that the entropy will increase. This is also true for more general quantum operations. Moreover, one may be interested in quantum entropies as signatures of an arrow of time: to this end one can see how the value of an entropic measure changes under the action of a general quantum operation. More concretely, let us consider general quantum operations represented by completely positive and trace-preserving maps $\mathcal{E}$, expressed in the Kraus form $\mathcal{E}(\rho)=\sum_{k=1}^{K} A_{k} \rho A_{k}^{\dagger}$ (with $\left\{A_{k}^{\dagger} A_{k}\right\}$ satisfying the completeness relation $\left.\sum_{k=1}^{K} A_{k}^{\dagger} A_{k}=I\right)$. It can be shown that the behavior of entropic measures depends nontrivially on the nature of the quantum operation (see e.g. [25, Sec. 12.6]). For example, a completely positive map increases the von Neumann entropy for every state if and only if it is bistochastic, i.e., if it is also unital ( $\left\{A_{k} A_{k}^{\dagger}\right\}$ also satisfies the completeness relation), so that the operation leaves the maximally mixed state invariant. This is no longer true for the case of a stochastic (but not bistochastic) quantum operation. What can be said of the generalized quantum $(h, \phi)$-entropies? This is summarized in the following:

Proposition 7 Let $\mathcal{E}$ be a bistochastic map. Then, the quantum operation $\rho \mapsto \mathcal{E}(\rho)$ can only degrade the information (i.e., increase the $(h, \phi)$-entropy):

$$
\mathbf{H}_{(h, \phi)}(\rho) \leq \mathbf{H}_{(h, \phi)}(\mathcal{E}(\rho))
$$

with equality if and only if $\mathcal{E}(\rho)=U \rho U^{\dagger}$ for a unitary operator $U$.

Proof From the quantum Hardy-Littlewood-Pólya theorem [25, Lemma. 12.1], $\mathcal{E}(\rho) \prec \rho$, so that the proposition is a consequence of the Schur concavity of the quantum $(h, \phi)$-entropy (Proposition 1$)$. Let us mention that an isometric operator can define a bistochastic map only if it is unitary.

This is a well-known property of von Neumann entropy, when dealing with projective measurements $A_{k} A_{l}=\delta_{k, l} A_{k}$ [3, Th. 11.9]. It turns out to be true for the whole family of $(h, \phi)$-entropies, and in a more general context than projective measurements. However, as we have noticed above, generalized (but not bistochastic) quantum operations can decrease the quantum $(h, \phi)$-entropy. Let us consider the example given in [3, Ex. 11.15, p. 515]. Let $\rho$ be the density operator of an arbitrary qubit system, with nonvanishing quantum $(h, \phi)$-entropy, and consider the generalized measurement performed by the measurement operators $A_{1}=|0\rangle\langle 0|$ and $A_{2}=|0\rangle\langle 1|$ (a completely positive map, but not unital). Then, the system after this measurement is represented by $\mathcal{E}(\rho)=|0\rangle\langle 0|\rho| 0\rangle\langle 0|+| 0\rangle\langle 1|\rho| 1\rangle\langle 0|=| 0\rangle\langle 0|$ with vanishing quantum $(h, \phi)$-entropy.

Note that Proposition 5 can be viewed as a consequence of Proposition 7. Indeed, it is straightforward to see that the set of operators $E=\left\{\left|e_{k}\right\rangle\left\langle e_{k}\right|\right\}$ defines the bistochastic $\operatorname{map} \mathcal{E}(\rho)=\sum_{k=1}^{N} p_{k}^{E}(\rho)\left|e_{k}\right\rangle\left\langle e_{k}\right|$. Thus, Proposition 5 can be deduced applying successively Propositions 7 and 4.

In the light of the previous discussions and results, we can reinterpret Proposition 5 as follows: the quantum $(h, \phi)$-entropy equals the minimum over the set of rank-one projective measurements of the classical $(h, \phi)$-entropy for a given measurement and 
density operator. Indeed, we can extend the minimization domain to the set of rankone positive operator valued measurements (POVMs). ${ }^{2}$ As a consequence, we can give an alternative (and very natural, from a physical perspective) definition for the $(h, \phi)$-entropies.

Proposition 8 Let $\mathbb{E}$ be the set of all rank-one POVMs. Then

$$
\mathbf{H}_{(h, \phi)}(\rho)=\min _{E \in \mathbb{E}} H_{(h, \phi)}\left(p^{E}(\rho)\right)
$$

where $p^{E}(\rho)$ is the probability vector for the POVME $=\left\{E_{k}\right\}_{k=1}^{K}$ given the density operator $\rho$, i.e., $p_{k}^{E}(\rho)=\operatorname{Tr}\left(E_{k} \rho\right)$.

Proof Let us consider an arbitrary rank-one POVM $E=\left\{E_{k}\right\}_{k=1}^{K}$ and consider the positive operators $A_{k}=A_{k}^{\dagger}=E_{k}^{\frac{1}{2}}$. Let us then define

$$
\mathcal{E}_{E}(\rho)=\sum_{k=1}^{K} E_{k}^{\frac{1}{2}} \rho E_{k}^{\frac{1}{2}}=\sum_{k=1}^{K} p_{k}^{E}(\rho) \frac{E_{k}^{\frac{1}{2}} \rho E_{k}^{\frac{1}{2}}}{\operatorname{Tr} E_{k}^{\frac{1}{2}} \rho E_{k}^{\frac{1}{2}}}=\sum_{k=1}^{K} p_{k}^{E}(\rho)\left|\psi_{k}\right\rangle\left\langle\psi_{k}\right|,
$$

where we have used the fact that $E_{k}$ is rank-one, so its square root can be written in the form $E_{k}^{\frac{1}{2}}=\left|\tilde{e}_{k}\right\rangle\left\langle\tilde{e}_{k}\right|$ (with $\left|\tilde{e}_{k}\right\rangle$ not necessarily normalized), allowing us to introduce the pure states $\left|\psi_{k}\right\rangle=\frac{\left|\tilde{e}_{k}\right\rangle}{\left\langle\tilde{e}_{k} \mid \tilde{e}_{k}\right\rangle^{\frac{1}{2}}}$. From the completeness relation satisfied by the POVM, $\mathcal{E}_{E}(\rho)$ is then a doubly stochastic map. Thus, applying successively Proposition 7 and Proposition 4 we obtain

$$
\mathbf{H}_{(h, \phi)}(\rho) \leq \mathbf{H}_{(h, \phi)}(\mathcal{E}(\rho)) \leq H_{(h, \phi)}\left(p^{E}(\rho)\right)
$$

Since $E$ is arbitrary, we thus have

$$
\mathbf{H}_{(h, \phi)}(\rho) \leq \min _{E \in \mathbb{E}} H_{(h, \phi)}\left(p^{E}(\rho)\right)
$$

Consider then $E_{\min }=\left\{\left|e_{k}\right\rangle\left\langle e_{k}\right|\right\}_{k=1}^{N}$ where $\left\{\left|e_{k}\right\rangle\right\}_{k=1}^{N}$ is the orthonormal basis that diagonalizes $\rho$. Thus

$$
H_{(h, \phi)}\left(p^{E_{\min }}(\rho)\right)=\mathbf{H}_{(h, \phi)}(\rho) \leq \min _{E \in \mathbb{E}} H_{(h, \phi)}\left(p^{E}(\rho)\right) \leq H_{(h, \phi)}\left(p^{E_{\min }}(\rho)\right),
$$

which ends the proof.

We notice that the alternative definition of quantum $(h, \phi)$-entropy given in this proposition cannot be extended to any POVM. The following counterexample shows this impossibility. Let us consider the density operator $\rho=\frac{I_{N}}{N}$ with $N>2$ even,

2 Recall that a POVM is a set $\left\{E_{k}\right\}$ of positive definite operators satisfying the resolution of the identity 
and the POVM $E=\left\{E_{1}, E_{2}\right\}$ formed by the positive operators $E_{1}=\sum_{i=1}^{\frac{N}{2}}\left|e_{i}\right\rangle\left\langle e_{i}\right|$ and $E_{2}=\sum_{i=\frac{N}{2}+1}^{N}\left|e_{i}\right\rangle\left\langle e_{i}\right|$, where $\left\{\left|e_{i}\right\rangle\right\}_{i=1}^{N}$ is an arbitrary orthonormal basis of $\mathcal{H}^{N}$. Thus, we obtain $p^{E}(\rho)=\left[\begin{array}{ll}\frac{1}{2} & \frac{1}{2}\end{array}\right]^{t}$ and consequently from the Schur concavity and the expansibility of the classical $(h, \phi)$-entropy we have $\mathbf{H}_{(h, \phi)}(\rho)=h\left(N \phi\left(\frac{1}{N}\right)\right)>$ $h\left(2 \phi\left(\frac{1}{2}\right)\right)=H_{(h, \phi)}\left(p^{E}(\rho)\right)$.

\subsection{Composite systems I: additivity, sub and superadditivities, and bipartite pure states}

We focus now on some properties of the quantum $(h, \phi)$-entropies for bipartite quantum systems $A B$ represented by density operators acting on a product Hilbert space $\mathcal{H}_{A B}=\mathcal{H}_{A}^{N_{A}} \otimes \mathcal{H}_{B}^{N_{B}}$. Specifically, we are interested in the behavior of the entropy of the composite density operator $\rho^{A B}$, with reference to the entropies of the density operators of the subsystems, ${ }^{3} \rho^{A}=\operatorname{Tr}_{B} \rho^{A B}$ and $\rho^{B}=\operatorname{Tr}_{A} \rho^{A B}$.

Now, we give sufficient conditions for the additivity property of quantum $(h, \phi)$ entropies:

Proposition 9 Let $\rho^{A} \otimes \rho^{B}$ be an arbitrary product density operator of a composite system $A B$, and $\rho^{A}$ and $\rho^{B}$ the corresponding density operators of the subsystems. If, for $(a, b) \in(0,1]^{2}$ and $(x, y) \in\left[\min \left\{\phi(1), N_{A} \phi\left(\frac{1}{N_{A}}\right)\right\}, \max \left\{\phi(1), N_{A} \phi\left(\frac{1}{N_{A}}\right)\right\}\right]$ $\times\left[\min \left\{\phi(1), N_{B} \phi\left(\frac{1}{N_{B}}\right)\right\}, \max \left\{\phi(1), N_{B} \phi\left(\frac{1}{N_{B}}\right)\right\}\right], \phi$ and $h$ satisfy the Cauchy functional equations either of the form (i) $\phi(a b)=\phi(a) b+a \phi(b)$ and $h(x+y)=$ $h(x)+h(y)$, or of the form (ii) $\phi(a b)=\phi(a) \phi(b)$ and $h(x y)=h(x)+h(y)$. Then the $(h, \phi)$-entropy satisfies the additivity property

$$
\mathbf{H}_{(h, \phi)}\left(\rho^{A} \otimes \rho^{B}\right)=\mathbf{H}_{(h, \phi)}\left(\rho^{A}\right)+\mathbf{H}_{(h, \phi)}\left(\rho^{B}\right)
$$

Proof In case (i), by writing the density operators $\rho^{A}$ and $\rho^{B}$ in their diagonal forms, it is straightforward to obtain

$$
\phi\left(\rho^{A} \otimes \rho^{B}\right)=\phi\left(\rho^{A}\right) \otimes \rho^{B}+\rho^{A} \otimes \phi\left(\rho^{B}\right),
$$

\footnotetext{
3 By definition, the partial trace operation over $B, \operatorname{Tr}_{B}: \mathcal{H}_{A}^{N_{A} \otimes} \mathcal{H}_{B}^{N_{B}} \rightarrow \mathcal{H}_{A}^{N_{A}}$, is the unique linear operator such that $\operatorname{Tr}_{B} X_{A} \otimes X_{B}=\left(\operatorname{Tr}_{B} X_{B}\right) X_{A}$ for all $X_{A}$ and $X_{B}$ acting on $\mathcal{H}_{A}^{N_{A}}$ and $\mathcal{H}_{B}^{N_{B}}$, respectively. For instance, let us consider the bases $\left\{\left|e_{i}^{A}\right\rangle\right\}_{i=1}^{N_{A}}$ and $\left\{\left|e_{j}^{B}\right\rangle\right\}_{j=1}^{N_{B}}$ of $\mathcal{H}_{A}^{N_{A}}$ and $\mathcal{H}_{B}^{N_{B}}$ respectively, and the product basis $\left\{\left|e_{i}^{A}\right\rangle \otimes\left|e_{j}^{B}\right\rangle\right\}$ of $\mathcal{H}_{A}^{N_{A}} \otimes \mathcal{H}_{B}^{N_{B}}$. Let us denote by $\rho_{i j, i^{\prime} j^{\prime}}^{A B}$ the components in the product basis of an operator $\rho^{A B}$ acting on $\mathcal{H}_{A}^{N_{A}} \otimes \mathcal{H}_{B}^{N_{B}}$. Thus, the partial trace over $B$ of $\rho^{A B}$ gives the density operator of the subsystem $A, \rho^{A}=\operatorname{Tr}_{B} \rho^{A B}$, whose components are $\rho_{i, i^{\prime}}^{A}=\sum_{j} \rho_{i j, i^{\prime} j}^{A B}$ in the basis $\left\{\left|e_{i}^{A}\right\rangle\right\}$.
} 
and thus

$$
\begin{aligned}
& h\left(\operatorname{Tr} \phi\left(\rho^{A} \otimes \rho^{B}\right)\right)=h\left(\operatorname{Tr} \phi\left(\rho^{A}\right)+\operatorname{Tr} \phi\left(\rho^{B}\right)\right) \\
& \quad=h\left(\operatorname{Tr} \phi\left(\rho^{A}\right)\right)+h\left(\operatorname{Tr} \phi\left(\rho^{B}\right)\right)
\end{aligned}
$$

where we used $\operatorname{Tr} \rho^{A}=1=\operatorname{Tr} \rho^{B}$. Similarly, for case (ii),

$$
\phi\left(\rho^{A} \otimes \rho^{B}\right)=\phi\left(\rho^{A}\right) \otimes \phi\left(\rho^{B}\right)
$$

and thus

$$
h\left(\operatorname{Tr} \phi\left(\rho^{A} \otimes \rho^{B}\right)\right)=h\left(\operatorname{Tr} \phi\left(\rho^{A}\right) \operatorname{Tr} \phi\left(\rho^{B}\right)\right)=h\left(\operatorname{Tr} \phi\left(\rho^{A}\right)\right)+h\left(\operatorname{Tr} \phi\left(\rho^{B}\right)\right) .
$$

The domains where the functional equations have to be satisfied are, respectively, the domain of definition of $\phi$ and the image of $\operatorname{Tr} \phi$ (see Proposition 2).

Note that, on the one hand, in case (i) the functional equation for $\phi$ can be recast as $g(a b)=g(a)+g(b)$ with $g(x)=x^{-1} \phi(x)$. Thus, $\phi(x)=c_{1} x \ln x$ and $h(x)=c_{2} x$ with $c_{1} c_{2}<0$ are entropic functionals that are solutions of the functional equations (i). ${ }^{4}$ These solutions lead to von Neumann entropy, which, as it is well known, is additive (see e.g. [56,57]). On the other hand, in case (ii), $\phi(x)=x^{\alpha}$ and $h(x)=c \ln x$ with $0<\alpha<1$ and $c>0$ or $\alpha>1$ and $c<0$ are entropic functional solutions. This is the case for the Rényi entropies, which are also known to be additive (see e.g. [62]). In general, however, $(h, \phi)$-entropies are not additive, for instance quantum unified entropies (including quantum Tsallis entropies) do not satisfy this property for all the possible values of the entropic parameters [13,53]. For the (not so general) quantum $(f, \alpha)$-entropies, we can give necessary and sufficient conditions for the additivity property:

Proposition 10 Let $\rho^{A} \otimes \rho^{B}$ be an arbitrary product density operator of a composite system $A B$, and $\rho^{A}$ and $\rho^{B}$ the corresponding density operators of the subsystems. Then, for any $\alpha>0$, the additivity property

$$
\mathbf{F}_{(f, \alpha)}\left(\rho^{A} \otimes \rho^{B}\right)=\mathbf{F}_{(f, \alpha)}\left(\rho^{A}\right)+\mathbf{F}_{(f, \alpha)}\left(\rho^{B}\right)
$$

holds if and only if $f(x y)=f(x)+f(y)$ for $(x, y) \in\left[\min \left\{1, N_{A}^{1-\alpha}\right\}, \max \left\{1, N_{A}^{1-\alpha}\right\}\right]$ $\times\left[\min \left\{1, N_{B}^{1-\alpha}\right\}, \max \left\{1, N_{B}^{1-\alpha}\right\}\right]$.

\footnotetext{
${ }^{4}$ Notice that the Cauchy equations $g(x+y)=g(x)+g(y), g(x y)=g(x)+g(y)$ and $g(x y)=g(x) g(y)$ are not necessarily linear, logarithmic or power type, respectively, without additional assumptions on the domain where they are satisfied and on the class of admissible functions (see e.g. [43,64]). But, recall that the entropic functionals $h$ and $\phi$ are continuous and either increasing and concave, or decreasing and convex.
} 
Proof The 'if' part is a direct consequence of Proposition 9 where $\phi(x)=x^{\alpha}$ and $h(x)=\frac{f(x)}{1-\alpha}$ satisfy the Cauchy equations of condition (ii).

Reciprocally, if $\mathbf{F}_{(f, \alpha)}$ is additive, we necessarily have that $f\left(\operatorname{Tr}\left(\rho^{A}\right)^{\alpha} \operatorname{Tr}\left(\rho^{B}\right)^{\alpha}\right)=$ $f\left(\operatorname{Tr}\left(\rho^{A}\right)^{\alpha}\right)+f\left(\operatorname{Tr}\left(\rho^{B}\right)^{\alpha}\right)$ for any pair of arbitrary states. Denoting $x=\operatorname{Tr}\left(\rho^{A}\right)^{\alpha}$ and $y=\operatorname{Tr}\left(\rho^{B}\right)^{\alpha}$ and analyzing the image of $\operatorname{Tr} \rho^{\alpha}$ for any density operator acting on $\mathcal{H}^{N}$, we necessarily have $f(x y)=f(x)+f(y)$ over the domain specified in the proposition, which ends the proof.

Notice that, if $f$ is twice differentiable, one can show that $f$ is proportional to the logarithm thus, among the quantum $(f, \alpha)$-entropies, only the von Neumann and quantum Rényi entropies are additive.

As we have seen, the $(h, \phi)$-entropies are, in general, nonadditive. However, as suggested in [65], two types of subadditivity and superadditivity can be of interest. One of them compares the entropy of $\rho^{A B}$ with the sum of the entropies of the subsystems $\rho^{A}$ and $\rho^{B}$ (global entropy vs sum of marginal-entropies), and the other one compares the entropy of $\rho^{A B}$ with that of the product state $\rho^{A} \otimes \rho^{B}$ (global entropy vs product-ofmarginals entropy). The general study of subadditivity of the first type, $\mathbf{H}_{(h, \phi)}\left(\rho^{A B}\right) \leq$ $\mathbf{H}_{(h, \phi)}\left(\rho^{A}\right)+\mathbf{H}_{(h, \phi)}\left(\rho^{B}\right)$, is difficult, even if one is looking for sufficient conditions to insure this subadditivity. Although it is not valid in general, there are certain cases for which it holds. For example, it holds for the von Neumann entropy [57], quantum unified entropies for a restricted set of parameters [13,53], and quantum Tsallis entropy with parameter greater than $1[65,66]$. On the other hand, it is possible to show that only the von Neumann entropy (or an increasing function of it) satisfies subadditivity of the second type, provided that some smoothness conditions are imposed on $\phi$. This is summarized in the following:

Proposition 11 Let $\rho^{A B}$ be a density operator of a composite system $A B$, and $\rho^{A}$ and $\rho^{B}$ the corresponding density operators of the subsystems. Assume that $\phi$ is twice differentiable on $(0,1)$. The $(h, \phi)$-entropy satisfies

$$
\mathbf{H}_{(h, \phi)}\left(\rho^{A B}\right) \leq \mathbf{H}_{(h, \phi)}\left(\rho^{A} \otimes \rho^{B}\right)
$$

if and only if $\mathbf{H}_{(h, \phi)}$ is an increasing function of the von Neumann entropy, given by $\phi(x)=-x \ln x$.

Proof The proof is based on two steps:

- First, an example of a two qutrit diagonal system acting on a Hilbert space $\mathcal{H}^{3} \otimes \mathcal{H}^{3}$ is presented, for which it is shown that $\mathbf{H}_{(h, \phi)}$ cannot be subadditive, with the exception of certain functions $\phi^{\prime}$ satisfying a given functional equation.

- Next, under the assumptions of the proposition, the functional equation is solved, and it is shown that all the entropic functionals $\phi$ for which we could not conclude on the subadditivity of $\mathbf{H}_{(h, \phi)}$, can be reduced to the case $\phi(x)=-x \ln x$ and $h$ increasing. 
Step 1 Consider the composite two qutrit systems acting on a Hilbert space $\mathcal{H}^{3} \otimes \mathcal{H}^{3}$, of the form

$$
\rho^{A B}=\rho^{A} \otimes \rho^{B}-c(|00\rangle\langle 00|+| 11\rangle\langle 11|-| 10\rangle\langle 10|-| 01\rangle\langle 01|)
$$

with

$$
\begin{aligned}
\rho^{A} & =a|0\rangle\langle 0|+\alpha| 1\rangle\langle 1|+(1-a-\alpha)| 2\rangle\langle 2| \\
\text { and } \quad \rho^{B} & =b|0\rangle\langle 0|+\beta| 1\rangle\langle 1|+(1-b-\beta)| 2\rangle\langle 2|
\end{aligned}
$$

where $\{|0\rangle,|1\rangle,|2\rangle\}$ is an orthonormal basis for $\mathcal{H}^{3},|i j\rangle=|i\rangle \otimes|j\rangle$, the coefficients $(a, \alpha, b, \beta)$ in the set

$$
D=\{a, \alpha, b, \beta: 0<a, b<1 \wedge 0<\alpha \leq 1-a \wedge 0<\beta \leq 1-b\}
$$

and $c$ in the interval

$$
C_{a, \alpha, b, \beta}=[-1+\max \{a b, \alpha \beta, 1-a \beta, 1-\alpha b\}, \min \{a b, \alpha \beta, 1-a \beta, 1-\alpha b\}]
$$

Let us now recall the Klein inequality [25, Eq. (12.7)] for concave $\phi$,

$$
\operatorname{Tr} \phi(\rho)-\operatorname{Tr} \phi(\sigma) \leq \operatorname{Tr}\left((\rho-\sigma) \phi^{\prime}(\sigma)\right)
$$

the reversed inequality holds for convex $\phi$. If the Klein inequality is applied to $\rho=$ $\rho^{A} \otimes \rho^{B}$ and $\sigma=\rho^{A B}$, for $(a, b) \in(0,1)^{2}$ (such that $C_{a, \alpha, b, \beta}$ is not restricted to $\{0\}$ ), and $c \in \stackrel{\circ}{C}_{a, \alpha, b, \beta}$ (where ${ }^{\circ}$ denotes the interior of a set), we obtain for concave $\phi$,

$$
\operatorname{Tr} \phi\left(\rho^{A} \otimes \rho^{B}\right)-\operatorname{Tr} \phi\left(\rho^{A B}\right) \leq c g(a, \alpha, b, \beta, c),
$$

and the reversed inequality for convex $\phi$, where

$$
g(a, \alpha, b, \beta, c)=\phi^{\prime}(a b-c)+\phi^{\prime}(\alpha \beta-c)-\phi^{\prime}(a \beta+c)-\phi^{\prime}(\alpha b+c) .
$$

Assume that there exists $(x, u, y, v) \in \stackrel{\circ}{D}$ such that $g(x, u, y, v, 0) \neq 0$. From the continuity of $\phi^{\prime}$, function $g$ is continuous, and thus there exists a neighborhood $V_{0} \subset$ $\stackrel{\circ}{C}_{x, u, y, v}$ of 0 such that function $c \mapsto g(x, u, y, v, c)$ has a constant sign on $V_{0}$. As a conclusion, $c \mapsto c g(x, u, y, v, c)$ does not preserve sign on $V_{0}$. This allows us to conclude from (23) that when $\phi$ is concave (resp. convex), $\operatorname{Tr} \phi\left(\rho^{A B}\right)$ can be higher (resp. lower) than $\operatorname{Tr} \phi\left(\rho^{A} \otimes \rho^{B}\right)$. Together with the increasing (resp. decreasing) property of $h$, it is then clear that if $g(a, \alpha, b, \beta, 0)$ is not identically zero on the domain $\stackrel{\circ}{D}$, then $\mathbf{H}_{(h, \phi)}$ cannot be subadditive in the sense global vs product of marginals.

Step 2 If $g(a, \alpha, b, \beta, 0)=0$ on $\stackrel{\circ}{D}$, then $\phi^{\prime}$ satisfies the functional equation

$$
\phi^{\prime}(a b)+\phi^{\prime}(\alpha \beta)-\phi^{\prime}(a \beta)-\phi^{\prime}(\alpha b)=0
$$


and one cannot use the previous argument to decide if $\mathbf{H}_{(h, \phi)}$ is subadditive or not. In order to solve this riddle we follow $[67, \S 6]$, where a similar functional equation is discussed. By fixing $(a, b) \in(0,1)^{2}$, differentiating identity (25) with respect to $\alpha$ and multiplying the result by $\alpha$, we obtain

$$
\alpha \beta \phi^{\prime \prime}(\alpha \beta)=\alpha b \phi^{\prime \prime}(\alpha b) \quad \text { for } \quad(\alpha, \beta) \in(0,1-a) \times(0,1-b) .
$$

This means that $x \phi^{\prime \prime}(x)$ is constant for $x \in(0,(1-a) \max \{b, 1-b\})$, for all $(a, b) \in$ $(0,1)^{2}$. Thus, $x \phi^{\prime \prime}(x)$ is constant for $x \in(0,1)$. In other words, $\phi$ is necessarily of the form $\phi(x)=-\lambda x \ln x+\mu x+v$. Due to the continuity of $\phi$, this is valid on the closed set $[0,1]$. Since $\operatorname{Tr} \rho=1$, one can restrict the analysis to $\mu=0$ (this value can be put in $\nu$, leaving the entropy unchanged). Moreover, this constant does not alter the concavity or convexity of $\phi$ and thus can be put in $h$ [without altering its monotonicity and, thus, the sense of the inequalities between $\mathbf{H}_{(h, \phi)}\left(\rho^{A B}\right)$ and $\mathbf{H}_{(h, \phi)}\left(\rho^{A} \otimes \rho^{B}\right)$ either]. To ensure strict concavity (convexity) of $\phi$, one must have $\lambda>0$ (resp. $\lambda<0$ ) and thus, without loss of generality, $\lambda$ can be rejected in $h$. Finally, one can rapidly see that $\phi(x)=-x \ln x$ satisfies the identity (25).

As a conclusion, under the assumptions of the proposition, when $\mathbf{H}_{(h, \phi)}$ is not an increasing function of the von Neumann entropy, it cannot be subadditive. Reciprocally, the von Neumann entropy is known to be subadditive (see e.g. [56,57]), and this remains valid for any increasing function of this entropy, which finishes the proof.

Notice that neither Rényi nor Tsallis entropies satisfy this subadditivity for any entropic parameter except for $\alpha=1^{5}$ (i.e., von Neumann entropy). As a consequence, from Propositions 10 and 11, we obtain that, except for the von Neumann case (and the zero-parameter entropy), Rényi entropies do not neither satisfy usual subadditivity in terms of $\mathbf{H}_{(h, \phi)}\left(\rho^{A B}\right)$ and $\mathbf{H}_{(h, \phi)}\left(\rho^{A}\right)+\mathbf{H}_{(h, \phi)}\left(\rho^{B}\right)$ (as in the classical counterpart [68, Ch. IX, §6]). In addition, the counterexample used in the proof of the proposition allows us to conclude that the same nonsubadditivity also holds for the classical counterpart of $(h, \phi)$-entropies.

Regarding both types of superadditivity, it is well known that the von Neumann entropy does not satisfy neither of them. Here, we extend this fact to any $(h, \phi)$-entropy, as summarized in the following:

Proposition 12 Let $\rho^{A B}$ be a density operator of a composite system $A B$, and $\rho^{A}$ and $\rho^{B}$ the corresponding density operators of the subsystems. The $(h, \phi)$-entropy is nonsuperadditive in the sense that

$$
\begin{aligned}
& \mathbf{H}_{(h, \phi)}\left(\rho^{A B}\right) \geq \mathbf{H}_{(h, \phi)}\left(\rho^{A} \otimes \rho^{B}\right) \quad \text { and } \\
& \mathbf{H}_{(h, \phi)}\left(\rho^{A B}\right) \geq \mathbf{H}_{(h, \phi)}\left(\rho^{A}\right)+\mathbf{H}_{(h, \phi)}\left(\rho^{B}\right)
\end{aligned}
$$

are not satisfied for all states.

\footnotetext{
5 For $\alpha=0$ this subadditivity is also satisfied, but note that in this special case, $\phi$ is not continuous and moreover does not fulfill the conditions of the proposition.
} 
Proof Let us consider the two qubit diagonal system acting on $\mathcal{H}^{2} \otimes \mathcal{H}^{2}$ :

$$
\rho^{A B}=\frac{1}{2}(|00\rangle\langle 00|+| 11\rangle\langle 11|),
$$

which gives $\rho^{A}=\frac{I_{2}}{2}=\rho^{B}$. In this case, we have $\mathbf{H}_{(h, \phi)}\left(\rho^{A B}\right)=h\left(2 \phi\left(\frac{1}{2}\right)\right)$, $\mathbf{H}_{(h, \phi)}\left(\rho^{A}\right)+\mathbf{H}_{(h, \phi)}\left(\rho^{B}\right)=2 h\left(2 \phi\left(\frac{1}{2}\right)\right)$ and $\mathbf{H}_{(h, \phi)}\left(\rho^{A} \otimes \rho^{B}\right)=h\left(4 \phi\left(\frac{1}{4}\right)\right)$, such that $\mathbf{H}_{(h, \phi)}\left(\rho^{A B}\right)<\mathbf{H}_{(h, \phi)}\left(\rho^{A}\right)+\mathbf{H}_{(h, \phi)}\left(\rho^{B}\right)$ (due to the positivity of the entropies) and $\mathbf{H}_{(h, \phi)}\left(\rho^{A B}\right)<\mathbf{H}_{(h, \phi)}\left(\rho^{A} \otimes \rho^{B}\right)$ (due to the Schur concavity property).

For the case of von Neumann entropy, it is well known that the entropy of subsystems of a bipartite pure state are equal (see e.g. [3, Th. 11.8-(3)],). We extend this result to any quantum $(h, \phi)$-entropy.

Proposition 13 Let $|\psi\rangle$ be a pure state of a composite system $A B$ and $\rho^{A}=$ $\operatorname{Tr}_{B}|\psi\rangle\langle\psi|$ and $\rho^{B}=\operatorname{Tr}_{A}|\psi\rangle\langle\psi|$ the corresponding density operators of the subsystems. Then

$$
\mathbf{H}_{(h, \phi)}\left(\rho^{A}\right)=\mathbf{H}_{(h, \phi)}\left(\rho^{B}\right) .
$$

Proof From the Schmidt decomposition theorem [25, Th. 9.1], any pure state $|\psi\rangle \in$ $\mathcal{H}_{A}^{N_{A}} \otimes \mathcal{H}_{B}^{N_{B}}$ can be written under the form

$$
|\psi\rangle=\sum_{i=1}^{N} \sqrt{\lambda_{i}}\left|e_{i}^{A}\right\rangle \otimes\left|e_{i}^{B}\right\rangle,
$$

where $\left\{\left|e_{i}^{A}\right\rangle\right\}_{i=1}^{N_{A}}$ and $\left\{\left|e_{i}^{B}\right\rangle\right\}_{i=1}^{N_{B}}$ are two orthonormal bases for $\mathcal{H}_{A}^{N_{A}}$ and $\mathcal{H}_{B}^{N_{B}}$, respectively, and $N \leq \min \left\{N_{A}, N_{B}\right\}$. The density operators of the subsystems are then

$$
\rho^{A}=\sum_{i=1}^{N} \lambda_{i}\left|e_{i}^{A}\right\rangle\left\langle e_{i}^{A}\right| \quad \text { and } \quad \rho^{B}=\sum_{i=1}^{N} \lambda_{i}\left|e_{i}^{B}\right\rangle\left\langle e_{i}^{B}\right|,
$$

so that the first $N$ eigenvalues $\lambda_{i}$ are equal, the remaining ones being zero. Therefore, using the expansibility property, both have the same quantum $(h, \phi)$-entropy.

\subsection{Composite systems II: entanglement detection}

Now, we consider the use of quantum $(h, \phi)$-entropies in the entanglement detection problem. As with the classical entropies, one would expect that the quantum entropies of density operators reduced to subsystems were lower than that of the density operator of the composite system. We show here that this property turns out to be valid for separable density operators. We recall that a bipartite quantum state is separable if it can be written as a convex combination of product states [69], that is, ${ }^{6}$

\footnotetext{
${ }^{6}$ Equivalently, the pure states $\left|\psi_{m}^{A}\right\rangle\left\langle\psi_{m}^{A}\right|$ and $\left|\psi_{m}^{B}\right\rangle\left\langle\psi_{m}^{B}\right|$ can be replaced by mixed states defined on $\mathcal{H}^{A}$ and $\mathcal{H}^{B}$, respectively [70].
} 


$$
\rho_{\text {Sep }}^{A B}=\sum_{m=1}^{M} \omega_{m}\left|\psi_{m}^{A}\right\rangle\left\langle\psi_{m}^{A}|\otimes| \psi_{m}^{B}\right\rangle\left\langle\psi_{m}^{B}\right| \quad \text { with } \quad \omega_{m} \geq 0 \text { and } \sum_{m=1}^{M} \omega_{m}=1 \text {. }
$$

For bipartite separable states, we have the following:

Proposition 14 Let $\rho_{\mathrm{Sep}}^{A B}$ be a separable density operator of a composite system $A B$, and let $\rho^{A}$ and $\rho^{B}$ be the corresponding density operators of the subsystems. Then

$$
\mathbf{H}_{(h, \phi)}\left(\rho_{\text {Sep }}^{A B}\right) \geq \max \left\{\mathbf{H}_{(h, \phi)}\left(\rho^{A}\right), \mathbf{H}_{(h, \phi)}\left(\rho^{B}\right)\right\},
$$

for any pair of entropic functionals $(h, \phi)$.

Proof This is a corollary of a more general criterion of separability given in Ref. [70] (also given in [25, B.4, p. 386]), based on majorization. Indeed, from that criterion, a separable density operator $\rho_{\text {Sep }}^{A B}$ and the reduced density operators $\rho^{A}$ and $\rho^{B}$ satisfy the majorization relations

$$
\rho_{\text {Sep }}^{A B} \prec \rho^{A} \quad \text { and } \quad \rho_{\text {Sep }}^{A B} \prec \rho^{B} \text {. }
$$

Inequality (32) is thus a consequence of the Schur concavity of $\mathbf{H}_{(h, \phi)}$, proved in Proposition 1.

It is worth mentioning that the majorization relations (33) do not imply the separability of the density operator [25,70] and thus (33) is a sufficient condition for the derivation of (32). In other words, some pair(s) of entropic functionals $(h, \phi)$ and a density operator of the composite system violate (32).

Proposition 14 was proved originally for von Neumann entropy [21], and later on for some other quantum entropies such as the Rényi, Tsallis, and Kaniadakis ones (see e.g. [12,22-24,27] or [25, B.5, p. 387]). Remarkably, this property turns out to be fulfilled by any quantum $(h, \phi)$-entropy.

As an example we use Proposition 14 in the case of $(f, \alpha)$-entropies, in order to verify its efficiency to detect entangled Werner states of two qubit systems. Werner density operators are of the form [69] or [25, Eq. (15.42), pp. 382-383]:

$$
\rho^{A B}=\omega\left|\Psi^{-}\right\rangle\left\langle\Psi^{-}\right|+(1-\omega) \frac{I_{4}}{4}
$$

where $\left|\Psi^{-}\right\rangle=\frac{1}{\sqrt{2}}(|00\rangle-|11\rangle)$ is the singlet state, $|0\rangle$ and $|1\rangle$ are eigenstates of the Pauli matrix $\sigma_{z}$, and $\omega \in[0,1]$. It is well known that Werner states are entangled if and only if $\omega>\frac{1}{3}$. The density operators of the subsystems are $\rho^{A}=\frac{I_{2}}{2}=\rho^{B}$. Therefore, following Proposition 14 for an $(f, \alpha)$-entropy, we can assert that the Werner states are entangled if the function

$$
Z_{f, \alpha}(\omega)= \begin{cases}\frac{f\left(3\left(\frac{1-\omega}{4}\right)^{\alpha}+\left(\frac{1+3 \omega}{4}\right)^{\alpha}\right)-f\left(2^{1-\alpha}\right)}{\alpha-1}, & \alpha \neq 1 \\ \frac{3(1-\omega) \ln (1-\omega)+(1+3 \omega) \ln (1+3 \omega)}{4}-\ln 2, & \alpha=1\end{cases}
$$






Fig. 1 Contour plot of function $Z_{\ln , \alpha}(\omega)$ versus $\omega$ and $\alpha$, as given in Eq. (35). To the right of the dashed line (at $\omega=\frac{1}{3}$ ), the Werner states (34) are entangled, while to its left they are separable. The solid line corresponds to $Z_{f, \alpha}(\omega)=0$ and limits two situations: to the right where $Z_{f, \alpha}(\omega)$ is positive and the criterion allows to conclude that the states are entangled, and to the left where nothing can be said about the states

is positive. Note that, since $f$ is increasing, the sign of $Z_{f, \alpha}$ does not depend on the choice of $f$, so that we can take $f(x)=\ln x$ without loss of generality. Figure 1 shows a contour plot of $Z_{\ln , \alpha}(\omega)$ versus $\omega$ and $\alpha$. The dashed line represents the boundary between the entangled situation $\left(\omega>\frac{1}{3}\right)$ and the separable one $\left(\omega<\frac{1}{3}\right)$, and the solid line distinguishes the situation $Z_{f, \alpha}(\omega)>0$ (to the right) from the situation $Z_{f, \alpha}(\omega)<0$ (to the left). It can be seen that, in this specific example, the entropic entanglement criterion is improved when $\alpha$ increases. This can be well understood noting that, when $\alpha \rightarrow \infty, Z_{\ln , \alpha}(\omega) \rightarrow \ln \left(\frac{1+3 \omega}{2}\right)$, that is positive if and only if $\omega>\frac{1}{3}$, i.e., if and only if the Werner states are entangled.

This simple illustration aims at showing that the use of a family of $(h, \phi)$-entropies instead of a particular one, or playing with the parameter(s) of parametrized $(h, \phi)$ entropies, allows one to improve entanglement detection.

Naturally the majorization entanglement criterion is stronger than the entropic one. Indeed, for the example given above, the majorization criterion detects all entangled Werner states. However, there are situations where the problem of computation of the eigenvalues of the density operator happens to be harder than the calculation of the trace in the entropy definition (at least for entropic functionals of the form $\phi(x)=x^{n}$, with $n$ integer). Moreover, from the converse of Karamata theorem (see Sect. 2), the majorization criterion becomes equivalent to the entropic one when considering the whole family of $(h, \phi)$-entropies. This allows us to expect that the more "nonequivalent" entropies are used, the better the entanglement detection should be.

Another motivation for the use of general entropies in entanglement detection is that, in a more realistic scenario, one does not have complete information about the 
density operator, so the majorization criterion, and consequently the entropic one can not be applied. It happens usually that one has partial information from mean values of certain observables. In that case, one needs to use some inference method to estimate the density operator compatible with the available information. One of the more common methods for obtaining the least biased density operator compatible with the actual information, is the maximum entropy principle [71] (MaxEnt for brevity). That is, the maximization of von Neumann entropy subject to the restrictions given by the observed data. However, this procedure can fail when dealing with composite systems, as shown in Ref. [72]. Indeed, MaxEnt using von Neumann entropy can lead to fake entanglement, which means that it predicts entanglement even when there exists a separable state compatible with the data. In Ref. [12] it was shown that, using concave quantum entropies of the form $\mathbf{H}_{(\mathrm{id}, \phi)}(\rho)=\operatorname{Tr}(\phi(\rho))$, it is possible to avoid fake entanglement when the partial information is given through Bell constraints.

Now we address the following question that arises in a natural way. Is it possible to use the constructions given above to say something about multipartite entanglement? As the number of subsystems grows, the entanglement detection problem becomes more and more involved, even for the simplest tripartite case (see e.g. [73-75]). Indeed, for a multipartite system, one has to distinguish between the so-called full separability and many types of partial separability (see e.g. [76] and references therein). Here, we briefly discuss a possible extension of Proposition 14 for fully separable states. The definition of full multipartite separability for $L$ subsystems acting on a Hilbert space $\mathcal{H}_{L}=\bigotimes_{l=1}^{L} \mathcal{H}_{l}^{N_{l}}$ is a direct extension of (31), that is,

$\rho_{\text {FullSep }}^{A_{1} \ldots A_{L}}=\sum_{m=1}^{M} \omega_{m}\left|\psi_{m}^{A_{1}}\right\rangle\left\langle\psi_{m}^{A_{1}}|\otimes \cdots \otimes| \psi_{m}^{A_{L}}\right\rangle\left\langle\psi_{m}^{A_{L}}\right|$, with $\omega_{m} \geq 0$ and $\sum_{m=1}^{M} \omega_{m}=1$.

For multipartite fully separable states, we have the following:

Proposition 15 Let $\rho_{\text {FullSep }}^{A_{1} \ldots A_{L}}$ be a fully separable density operator of an L-partite system, and let $\rho^{A_{1}}, \ldots, \rho^{A_{L}}$ be the corresponding density operators of the subsystems. Then

$$
\mathbf{H}_{(h, \phi)}\left(\rho_{\text {FullSep }}^{A_{1} \ldots A_{L}}\right) \geq \max \left\{\mathbf{H}_{(h, \phi)}\left(\rho^{A_{1}}\right), \ldots, \mathbf{H}_{(h, \phi)}\left(\rho^{A_{L}}\right)\right\}
$$

for any pair of entropic functionals $(h, \phi)$.

Proof The majorization relations (33) for separable bipartite states are mainly based on the Schrödinger mixture theorem, so that (33) can be generalized to the multipartite case in a direct way [70]. Let us consider the fully separable density operator (36), written in a diagonal form as $\rho_{\text {FullSep }}^{A_{1} \ldots A_{L}}=\sum_{i} \lambda_{i}\left|e_{i}\right\rangle\left\langle e_{i}\right|$. On the one hand, from the Schrödinger mixture theorem, there exists a unitary matrix $U$ such that

$$
\sqrt{\lambda_{i}}\left|e_{i}\right\rangle=\sum_{m} U_{i m} \sqrt{\omega_{m}}\left|\psi_{m}^{A_{1}}\right\rangle \otimes \cdots \otimes\left|\psi_{m}^{A_{l}}\right\rangle \otimes \cdots \otimes\left|\psi_{m}^{A_{L}}\right\rangle
$$

On the other hand, let $\rho^{A_{l}}=\sum_{m} \omega_{m}\left|\psi_{m}^{A_{l}}\right\rangle\left\langle\psi_{m}^{A_{l}}\right|$ be the density operator of the $l$ th subsystem and its diagonal form $\rho^{A_{l}}=\sum_{j} \lambda_{j}^{l}\left|e_{j}^{l}\right\rangle\left\langle e_{j}^{l}\right|$. Using again the Schrödinger 
mixture theorem, there is a unitary matrix $V^{l}$ such that

$$
\sqrt{\omega_{m}}\left|\psi_{m}^{A_{l}}\right\rangle=\sum_{j} V_{m j}^{l} \sqrt{\lambda_{j}^{l}}\left|e_{j}^{l}\right\rangle
$$

Substituting (39) into (38), left-multiplying the result by its adjoint and using the orthonormality, $\left\langle e_{j}^{l} \mid e_{j^{\prime}}^{l}\right\rangle=\delta_{j j^{\prime}}$ and $\left\langle e_{i} \mid e_{i^{\prime}}\right\rangle=\delta_{i i^{\prime}}$, we obtain

$$
\lambda=B^{l} \lambda^{l}
$$

with $B_{i j}^{l}=\sum_{m, m^{\prime}} U_{i m^{\prime}}^{*} U_{i m} V_{m^{\prime} j}^{l *} V_{m j}^{l} \prod_{l^{\prime} \neq l}\left\langle\psi_{m}^{A_{l^{\prime}}} \mid \psi_{m^{\prime}}^{A_{l^{\prime}}}\right\rangle$. Following similar arguments as in the bipartite case [70, Th. 1], it is straightforward to show that $B^{l}$ is a bistochastic matrix and thus that $\lambda \prec \lambda^{l}$. Finally, using the Schur concavity of $\mathbf{H}_{(h, \phi)}$, we obtain $\mathbf{H}_{(h, \phi)}\left(\rho_{\text {FullSep }}^{A_{1} \ldots A_{L}}\right) \geq \mathbf{H}_{(h, \phi)}\left(\rho^{A_{l}}\right)$ for any $l$, and thus inequality (37).

Some interesting problems to be addressed are the application of this proposition to particular multipartite states, as well as the extension of the generalized entropic criteria to different types of partial separability. These points and related derivations are beyond the scope of the present contribution and will be the subject of future research.

\section{On possible further generalized informational quantities}

How to obtain useful conditional entropies and mutual informations based on generalized entropies is an open question and there is no general consensus to answer it, even in the classical case (see e.g. [52,77-79] for different proposals). Here, we first discuss briefly two possible definitions of classical conditional entropies and mutual informations, based on $(h, \phi)$-entropies. We then proceed to obtain quantum versions of those quantities.

\subsection{A generalization of classical conditional entropies and mutual informations}

Let us consider a pair of random variables $(A, B)$ with joint probability vector $p^{A B}$, i.e., $p_{a, b}^{A B}=\operatorname{Pr}[A=a, B=b]$, and let us denote by $p^{A}$ and $p^{B}$ the corresponding marginal probability vectors, namely $p_{a}^{A}=\sum_{b} p_{a, b}^{A B}$ and $p_{b}^{B}=\sum_{a} p_{a, b}^{A B}$. From Bayes rule, the conditional probability vector for $A$ given that $B=b, p^{A \mid b}$, is defined by $p_{a}^{A \mid b}=\frac{p_{a, b}^{A B}}{p_{b}^{B}}$ (and analogously for $\left.p^{B \mid a}\right)$. For the sake of convenience, in this section, we indifferently denote $H_{(h, \phi)}(A, B)$ or $H_{(h, \phi)}\left(p^{A B}\right)$ (and similarly for the marginals).

In order to define a conditional $(h, \phi)$-entropy of $A$ given $B$, one possibility is to take the average of the $(h, \phi)$-entropy of the conditional probability $p^{A \mid b}$ over all outcomes for $B$ (in a way similar to the Shannon entropy [36], or also to the Rényi and Tsallis entropies, as in Refs. [78,79]). This leads to the following: 
Definition 3 Let us consider a pair of random variables $(A, B)$ with joint probability vector $p^{A B}$. We define the $\mathcal{J}$-conditional $(h, \phi)$-entropy of $A$ given $B$ as

$$
H_{(h, \phi)}^{\mathcal{J}}(A \mid B)=\sum_{b} p_{b}^{B} H_{(h, \phi)}\left(p^{A \mid b}\right)
$$

From Definition 3, one can thus define a " $\mathcal{J}$-mutual information" as

$$
\mathcal{J}_{(h, \phi)}(A ; B)=H_{(h, \phi)}(A)-H_{(h, \phi)}^{\mathcal{J}}(A \mid B)
$$

However, except when $h$ is concave, there is no guarantee that $\mathcal{J}_{(h, \phi)}(A ; B)$ is nonnegative (see Proposition 3). Besides, this quantity is not symmetric in general.

An alternative proposal is to mimic the chain rule satisfied by Shannon entropy, $H(A \mid B)=H(A, B)-H(B)$, and thus to define a conditional entropy as follows:

Definition 4 Let us consider a pair of random variables $(A, B)$ with joint probability vector $p^{A B}$. We define the $\mathcal{I}$-conditional $(h, \phi)$-entropy of $A$ given $B$ as

$$
H_{(h, \phi)}^{\mathcal{I}}(A \mid B)=H_{(h, \phi)}(A, B)-H_{(h, \phi)}(B) .
$$

It can be shown that this quantity is nonnegative from Petrović inequality [43, Th. 8.7.1] together with the appropriate increasing or decreasing behavior of $h$ (see also the properties of the $(h, \phi)$-entropies, Sect. 2).

From Definition 4, one can define a symmetric " $\mathcal{I}$-mutual information" as

$$
\mathcal{I}_{(h, \phi)}(A ; B)=H_{(h, \phi)}(A)-H_{(h, \phi)}^{\mathcal{I}}(A \mid B)=H_{(h, \phi)}(A)+H_{(h, \phi)}(B)-H_{(h, \phi)}(A, B) .
$$

Notice that $\mathcal{J}_{(h, \phi)}(A ; B)$ and $\mathcal{I}_{(h, \phi)}(A ; B)$ coincide in the Shannon case, i.e., for $h(x)=x, \phi(x)=-x \ln x$, but they are different in general. Besides, like $\mathcal{J}_{(h, \phi)}(A ; B), \mathcal{I}_{(h, \phi)}(A ; B)$ can also be negative.

Other alternatives have been proposed in specific contexts, such as for Tsallis [52, $77,78]$ or Rényi entropies [62,79-81], but a unified point of view is still missing and the question remains open.

\subsection{From classical to quantum generalized conditional entropies and mutual informations}

Let $\rho^{A B}$ be the density operator of a quantum composite system $A B$, and let $\Pi^{B}=$ $\left\{\Pi_{j}^{B}\right\}$ be a local projective measurement acting on $\mathcal{H}^{N_{B}}$, i.e., $\Pi_{j}^{B} \Pi_{j^{\prime}}^{B}=\delta_{j j^{\prime}} \Pi_{j}^{B}$ and $\sum_{j=1}^{N_{B}} \Pi_{j}^{B}=I_{N_{B}}$. The density operator relative to that measurement is given by $\rho^{A \mid \Pi^{B}}=\sum_{j} p_{j} \rho^{A \mid \Pi_{j}^{B}}$, where $p_{j}=\operatorname{Tr}\left(I \otimes \Pi_{j}^{B} \rho^{A B}\right)$ and $\rho^{A \mid \Pi_{j}^{B}}=\frac{I \otimes \Pi_{j}^{B} \rho^{A B} I \otimes \Pi_{j}^{B}}{\operatorname{Tr}\left(I \otimes \Pi_{j}^{B} \rho^{A B}\right)}$. 
We define the quantum version of (41) for $A$ conditioned to the local projective measurement $\Pi^{B}$ acting on $B$, as

$$
\mathbf{H}_{(h, \phi)}^{\mathcal{J}}\left(A \mid B_{\Pi^{B}}\right)=\sum_{j} p_{j} \mathbf{H}_{(h, \phi)}\left(\rho^{A \mid \Pi_{j}^{B}}\right) .
$$

This quantity has been proposed for trace-form entropies in [82]. One can obtain an independent quantum conditional entropy taking the minimum over the set of local projective measurement in (45), which leads to the following:

Definition 5 Let $\rho^{A B}$ be the density operator of a quantum composite system $A B$, and let $\Pi^{B}$ be a local projective measurement acting on $B$. We define the quantum $\mathcal{J}$-conditional $(h, \phi)$-entropy of $A$ given $B$ as

$$
\mathbf{H}_{(h, \phi)}^{\mathcal{J}}(A \mid B)=\min _{\left\{\Pi^{B}\right\}} \mathbf{H}_{(h, \phi)}^{\mathcal{J}}\left(A \mid B_{\Pi^{B}}\right)
$$

Now, we propose the quantum version of the mutual information (42) as

$$
\mathcal{J}_{(h, \phi)}(A ; B)=\mathbf{H}_{(h, \phi)}\left(\rho^{A}\right)-\mathbf{H}_{(h, \phi)}^{\mathcal{J}}(A \mid B) .
$$

Notice that if $h$ is concave, then $\mathcal{J}_{(h, \phi)} \geq 0$, but its nonnegativity is not guaranteed in general (see Proposition 3).

Another possibility is to extend the standard definition of the quantum conditional entropy to $(h, \phi)$-entropies, which leads to the quantum version of (43):

Definition 6 Let $\rho^{A B}$ be the density operator of a quantum composite system $A B$, and $\rho^{B}$ be the corresponding density operator of subsystem $B$. We define the quantum $\mathcal{I}$-conditional $(h, \phi)$-entropy of $A$ given $B$ as

$$
\mathbf{H}_{(h, \phi)}^{\mathcal{I}}(A \mid B)=\mathbf{H}_{(h, \phi)}\left(\rho^{A B}\right)-\mathbf{H}_{(h, \phi)}\left(\rho^{B}\right) .
$$

Notice that, contrary to the classical case, these quantities are not necessarily positive, except when $\rho^{A B}$ is separable, as we have precisely shown in Proposition 14.

On the other hand, one can propose a quantum version of (44), as follows

$$
\mathcal{I}_{(h, \phi)}(A ; B)=\mathbf{H}_{(h, \phi)}\left(\rho^{A}\right)+\mathbf{H}_{(h, \phi)}\left(\rho^{B}\right)-\mathbf{H}_{(h, \phi)}\left(\rho^{A B}\right) .
$$

Note, however, that there is no guarantee of nonnegativity of these quantities in general (see discussion before Proposition 12).

Unlike the classical case, the quantum mutual informations (47) and (49) are different even for the von Neumann case, and this difference is precisely the origin for the notion of quantum discord [83]. However, attempting to extend the definition of quantum discord through a direct replacement of von Neumann entropy by a generalized $(h, \phi)$-entropy fails in general (see e.g. [84,85]).

Unfortunately, all the quantities given in this section remain as formal definitions until one does not provide a complete study of their properties. This task lies beyond 
the scope of the present work and is currently under investigation [60] (see [86] for a different approach of the development of quantum information measures).

\section{Concluding remarks}

We have proposed a quantum version of the $(h, \phi)$-entropies first introduced by Salicrú et al. for classical systems. Along Sect. 3 we have presented our main results and definitions. Indeed, after the definition of quantum $(h, \phi)$-entropy given in Eq. (5), we have derived many basic properties related to Schur concavity and majorization, valid for any pair of entropic functionals $h$ and $\phi$ with the proper continuity, monotonicity and concavity properties established in Definition 2. Next, we have discussed the properties of the $(h, \phi)$-entropies in connection with the action of quantum operations and measurements. And later we have extended our study for the case of composite systems, focusing on important properties like additivity and subadditivity, and discussing an application to entanglement detection. Besides, in Sect. 4, an attempt to deal with generalized conditional entropies and mutual informations was presented, although it deserves deeper development, which is beyond our present scope.

The first advantage of our general approach has to do with the fact that many properties of particular examples of interest (such as von Neumann and quantum Rényi and Tsallis entropies) can be studied from the perspective of a unifying formal framework, which explains in an elegant fashion many of their common properties. In particular, our analysis reveals that majorization plays a key role in explaining most of the common features of the more important quantum entropic measures.

Remarkably enough, we have shown that many physical properties of the known quantum entropies, such as preservation under unitary evolution, and being nondecreasing under bistochastic quantum operations, hold in the general case. This is a signal indicating that our proposal yields new entropy functions which may be of interest for physical purposes. This kind of study can also be of use in applications to the description of quantum correlations, as we have seen in Sect. 3.5 for the case of bipartite entanglement; in the case of multipartite systems, we have also discussed a possible extension of the entropic entanglement criterion, however restricted to fully separable states. Finally, we mention that the present proposal may also have applications to quantum information processing and the problem of quantum state determination, because it allows for a more general systematization of the study of quantum entropies.

Acknowledgments GMB, FH, MP and PWL acknowledge CONICET and UNLP (Argentina), and MP and PWL also acknowledge SECyT-UNC (Argentina) for financial support. SZ is grateful to the University of Grenoble-Alpes (France) for the AGIR financial support.

\section{References}

1. Jozsa, R., Schumacher, B.: A new proof of the quantum noiseless coding theorem. J. Mod. Opt. 41(12), 2343 (1994). doi:10.1080/09500349414552191

2. Schumacher, B.: Quantum coding. Phys. Rev. A 51(4), 2738 (1995). doi:10.1103/PhysRevA.51.2738 
3. Nielsen, M.A., Chuang, I.L.: Quantum Computation and Quantum Information, 10th edn. Cambridge University Press, Cambridge (2010)

4. Renes, J.M.: The physics of quantum information: complementary uncertainty, and entanglement. Int. J. Quantum Inf. 11, 1330002 (2013). doi:10.1142/S0219749913300027

5. Ogawa, T., Hayashi, M.: On error exponents in quantum hypothesis testing. IEEE Trans. Inf. Theory 50(6), 1368 (2004). doi:10.1109/TIT.2004.828155

6. Holevo, A.: Probabilistic and Statistical Aspects of Quantum Theory. Quaderni Monographs, vol. 1, 2nd edn. Edizioni Della Normale, Pisa (2011)

7. Gill, R.D., Guţă, M.I.: On asymptotic quantum statistical inference. In: Banerjee, M., Bunea, F., Huang, J., Koltchinskii, V., Maathuis, M.H. (eds.) From Probability to Statistics and Back: High-Dimensional Models and Processes-A Festschrift in Honor of Jon A. Wellner, vol. 9, pp. 105-127. Institute of Mathematical Statistics collections, Beachwood, Ohio, USA (2013). doi:10.1214/12-IMSCOLL909

8. Yu, N., Duang, R., Ying, M.: Distinguishability of quantum states by positive operator-valued measures with positive partial transpose. IEEE Trans. Inf. Theory 60(4), 2069 (2004). doi:10.1109/TIT.2014. 2307575

9. von Neumann, J.: Thermodynamik quantenmechanischer Gesamtheiten. Nachrichten von der Gesellschaft der Wissenschaften zu Göttingen, pp. 273-291 (1927)

10. Rényi, A.: On measures of entropy and information. In: Proceedings of the 4th Berkeley Symposium on Mathematical Statistics and Probability, vol. 1, p. 547 (1961)

11. Tsallis, C.: Possible generalization of Boltzmann-Gibbs statistics. J. Stat. Phys. 52(1-2), 479 (1988). doi:10.1007/BF01016429

12. Canosa, N., Rossignoli, R.: Generalized nonadditive entropies and quantum entanglement. Phys. Rev. Lett. 88(17), 170401 (2002). doi:10.1103/PhysRevLett.88.170401

13. Hu, X., Ye, Z.: Generalized quantum entropy. J. Math. Phys. 47(2), 023502 (2006). doi:10.1063/1. 2165794

14. Kaniadakis, G.: Statistical mechanics in the context of special relativity. Phys. Rev. E 66(5), 056125 (2002). doi:10.1103/PhysRevE.66.056125

15. Maassen, H., Uffink, J.B.M.: Generalized entropic uncertainty relations. Phys. Rev. Lett. 60(12), 1103 (1988). doi:10.1103/PhysRevLett.60.1103

16. Uffink, J.B.M.: Measures of uncertainty and the uncertainty principle. Ph.D. thesis, University of Utrecht, Utrecht, The Netherlands (1990). See also references therein

17. Wehner, S., Winter, A.: Entropic uncertainty relations-a survey. New J. Phys. 12, 025009 (2010). doi:10.1088/1367-2630/12/2/025009

18. Zozor, S., Bosyk, G.M., Portesi, M.: On a generalized entropic uncertainty relation in the case of the qubit. J. Phys. A 46(46), 465301 (2013). doi:10.1088/1751-8113/46/46/465301

19. Zozor, S., Bosyk, G.M., Portesi, M.: General entropy-like uncertainty relations in finite dimensions. J. Phys. A 47(49), 495302 (2014). doi:10.1088/1751-8113/47/49/495302

20. Zhang, J., Zhang, Y., Yu, C.S.: Rényi entropy uncertainty relation for successive projective measurements. Quantum Inf. Process. 14(6), 2239 (2015). doi:10.1007/s11128-015-0950-z

21. Horodecki, R., Horodecki, P.: Quantum redundancies and local realism. Phys. Lett. A 194(3), 147 (1994). doi:10.1016/0375-9601(94)91275-0

22. Abe, S., Rajagopal, A.K.: Nonadditive conditional entropy and its significance for local realism. Phys. A 289(1-2), 157 (2001). doi:10.1016/S0378-4371(00)00476-3

23. Tsallis, C., Lloyd, S., Baranger, M.: Peres criterion for separability through nonextensive entropy. Phys. Rev. A 63(4), 042104 (2001). doi:10.1103/PhysRevA.63.042104

24. Rossignoli, R., Canosa, N.: Violation of majorization relations in entangled states and its detection by means of generalized entropic forms. Phys. Rev. A 67(4), 042302 (2003). doi:10.1103/PhysRevA.67. 042302

25. Bengtsson, I., Życzkowski, K.: Geometry of Quantum States: An Introduction to Quantum Entanglement. Cambridge University Press, Cambridge (2006)

26. Huang, Y.: Entanglement detection: complexity and Shannon entropic criteria. IEEE Trans. Inf. Theory 59(10), 6774 (2013). doi:10.1109/TIT.2013.2257936

27. Ourabah, K., Hamici-Bendimerad, A., Tribeche, M.: Quantum entanglement and Kaniadakis entropy. Phys. Scr. 90(4), 045101 (2015). doi:10.1088/0031-8949/90/4/045101

28. Yeung, R.W.: A framework for linear information inequalities. IEEE Trans. Inf. Theory 43(6), 1924 (1997). doi:10.1109/18.641556 
29. Zhang, Z., Yeung, R.W.: On characterization of entropy function via information inequalities. IEEE Trans. Inf. Theory 44(4), 1440 (1998). doi:10.1109/18.681320

30. Cardy, J.: Some results on the mutual information of disjoint regions in higher dimensions. J. Phys. A 28, 285402 (2013). doi:10.1088/1751-8113/46/28/285402

31. Gross, D., Walter, M.: Stabilizer information inequalities from phase space distributions. J. Math. Phys. 54(8), 082201 (2013). doi:10.1063/1.4818950

32. Wilde, M.M., Datta, N., Hsieh, M., Winter, A.: Quantum rate-distortion coding with auxiliary resources. IEEE Trans. Inf. Theory 59(10), 6755 (2013). doi:10.1109/TIT.2013.2271772

33. Datta, N., Renes, J.M., Renner, R., Wilde, M.M.: One-shot lossy quantum data compression. IEEE Trans. Inf. Theory 59(12), 8057 (2013). doi:10.1109/TIT.2013.2283723

34. Ahlswede, R., Löber, P.: Quantum data processing. IEEE Trans. Inf. Theory 47(1), 474 (2001). doi: 10. 1109/18.904565

35. Salicrú, M., Menéndez, M.L., Morales, D., Pardo, L.: Asymptotic distribution of $(h, \phi)$-entropies. Commun. Stat. Theory Methods 22(7), 2015 (1993). doi:10.1080/03610929308831131

36. Shannon, C.E.: A mathematical theory of communication. Bell Syst. Tech. J. 27, 623 (1948)

37. Havrda, J., Charvát, F.: Quantification method of classification processes: concept of structural $\alpha$ entropy. Kybernetika 3(1), 30 (1967)

38. Daróczy, Z.: Generalized information functions. Inf. Control 16(1), 36 (1970)

39. Rathie, P.N.: Unified $(r, s)$-entropy and its bivariate measures. Inf. Sci. 54(1-2), 23 (1991). doi:10. 1016/0020-0255(91)90043-T

40. Burbea, J., Rao, C.R.: On the convexity of some divergence measures based on entropy functions. IEEE Trans. Inf. Theory 28(3), 489 (1982). doi:10.1109/TIT.1982.1056497

41. Li, Y., Busch, P.: Von Neumann entropy and majorization. J. Math. Anal. Appl. 408(1), 384 (2013). doi:10.1016/j.jmaa.2013.06.019

42. Csiszàr, I.: Information-type measures of difference of probability distributions and indirect observations. Studia Scientiarum Mathematicarum Hungarica 2, 299 (1967)

43. Kuczma, M.: An Introduction to the Theory of Functional Equations and Inequalities: Cauchy's Equation and Jensen's Inequality, 2nd edn. Birkhäuser, Basel (2009)

44. Marshall, A.W., Olkin, I., Arnold, B.C.: Inequalities: Theory of Majorization and Its Applications, 2nd edn. Springer, New-York (2011). doi:10.1007/978-0-387-68276-1

45. Karamata, J.: Sur une inegalité relative aux fonctions convexes. Publications Mathématiques de l'Université de Belgrade 1, 145 (1932)

46. Bhatia, R.: Matrix Analysis. Springer, New-York (1997)

47. Khinchin, A.I.: Mathematical Foundations of Information Theory. Dover Publications, New-York (1957)

48. Tempesta, P.: Beyond the Shannon-Khinchin formulation: the composability axiom and the universalgroup entropy. Ann. Phys. 365, 180 (2016). doi:10.1016/j.aop.2015.08.013

49. Fadeev, D.K.: On the concept of entropy of a finite probabilistic scheme (Russian). Uspekhi Matematicheskikh Nauk 11(1(67)), 227 (1956)

50. Tsallis, C.: Introduction to Nonextensive Statistical Mechanics-Approaching a Complex World. Springer, New-York (2009). doi:10.1007/978-0-387-85359-8

51. Tempesta, P.: Formal groups and Z-entropies. arXiv preprint arXiv:1507.07436 (2016)

52. Rastegin, A.E.: Rényi and Tsallis formulations of noise-disturbance trade-off relations. Quantum Inf. Comput. 16(3\&4), 0313 (2016)

53. Rastegin, A.E.: Some general properties of unified entropies. J. Stat. Phys. 143(6), 1120 (2011). doi:10. 1007/s10955-011-0231-X

54. Fan, Y.J., Cao, H.X.: Monotonicity of the unified quantum $(r, s)$-entropy and $(r, s)$-mutual information. Quantum Inf. Process. 14(12), 4537 (2015). doi:10.1007/s11128-015-1126-6

55. Sharma, N.: Equality conditions for the quantum $f$-relative entropy and generalized data processing inequalities. Quantum Inf. Process. 11(1), 137 (2012). doi:10.1007/s11128-011-0238-x

56. Lieb, E.H.: Some convexity and subadditvity properties of entropy. Bull. Am. Math. Soc. 81(1), 1 (1975)

57. Wehrl, A.: General properties of entropies. Rev. Mod. Phys. 50(2), 221 (1978). doi:10.1103/ RevModPhys.50.221

58. Ohya, M., Petz, D.: Quantum Entropy and Its Use. Springer, Berlin (1993)

59. Lamberti, P.W., Portesi, M., Sparacino, J.: Natural metric for quantum information theory. Int. J. Quantum Inf. 7(5), 1009 (2009). doi:10.1142/S0219749909005584 
60. Bosyk, G.M., Bellomo, G., Zozor, S., Portesi, M., Lamberti, P.W.: Unified entropic measures of quantum correlations induced by local measurements. arXiv preprint arXiv:1604.00329 (2016)

61. Nielsen, M.A.: Probability distributions consistent with a mixed state. Phys. Rev. A 62, 052308 (2000). doi:10.1103/PhysRevA.62.052308

62. Müller-Lennert, M., Dupuis, F., Szehr, O., Fehr, S., Tomamichel, M.: On quantum Rényi entropies: a new generalization and some properties. J. Math. Phys. 54(12), 122203 (2013). doi:10.1063/1.4838856

63. Halmos, P.R.: A Hilbert Space Problem Book, 2nd edn. Springer, New-York (1982)

64. Cauchy, A.L.: Cours d'analyse de l'école royale polytechnique, vol. 1: analyse algébrique (Imprimerie royale (digital version, Cambrige, 2009), Paris, 1821)

65. Raggio, G.A.: Properties of $q$-entropies. J. Math. Phys. 36(9), 4785 (1995). doi:10.1063/1.530920

66. Audenaert, K.M.R.: Subadditivity of $q$-entropies for $q>1$. J. Math. Phys. 48(8), 083507 (2007). doi: $10.1063 / 1.2771542$

67. Daróczy, Z., Járai, A.: On the measurable solution of a functional equation arising in information theory. Acta Mathematica Academiae Scientiarum Hungaricae 34(1-2), 105 (1979). doi:10.1007/bf01902599

68. Rényi, A.: Probability Theory. North-Holland Publishing Company, Amsterdand (1970)

69. Werner, R.F.: Quantum states with Einstein-Podolsky-Rosen correlations admitting a hidden-variable model. Phys. Rev. A 40(8), 4277 (1989). doi:10.1103/PhysRevA.40.4277

70. Nielsen, M.A., Kempe, J.: Separable states are more disordered globally than locally. Phys. Rev. Lett. 86(22), 5184 (2001). doi:10.1103/PhysRevLett.86.5184

71. Jaynes, E.T.: Information theory and statistical mechanics. Phys. Rev. 106(4), 620 (1957). doi:10.1103/ PhysRev.106.620

72. Horodecki, R., Horodecki, M., Horodecki, P.: Entanglement processing and statistical inference: the Jaynes principle can produce fake entanglement. Phys. Rev. A 59(3), 1799 (1999). doi:10.1103/ PhysRevA.59.1799

73. Svetlichny, G.: Distinguishing three-body from two-body nonseparability by a Bell-type inequality. Phys. Rev. D 35(10), 3066 (1987). doi:10.1103/PhysRevD.35.3066

74. Mermin, N.D.: Simple unified form for the major no-hidden-variables theorems. Phys. Rev. Lett. 65(27), 3373 (1990). doi:10.1103/PhysRevLett.65.3373

75. Seevinck, M., Svetlichny, G.: Bell-type inequalities for partial separability in $N$-particle systems and quantum mechanical violations. Phys. Rev. Lett. 89(6), 060401 (2002). doi:10.1103/PhysRevLett.89. 060401

76. Horodecki, R., Horodecki, P., Horodecki, M., Horodecki, K.: Quantum entanglement. Rev. Mod. Phys. 81, 865 (2009). doi:10.1103/RevModPhys.81.865

77. Furuichi, S.: Information theoretical properties of Tsallis entropies. J. Math. Phys. 47(2), 023302 (2006). doi:10.1063/1.2165744

78. Rastegin, A.E.: Convexity inequalities for estimating generalized conditional entropies from below. Kybernetika 48(2), 242 (2012). http://eudml.org/doc/246939

79. Teixeira, A., Matos, A., Antunes, L.: Conditional Rényi entropies. IEEE Trans. Inf. Theory 58(7), 4272 (2012). doi:10.1109/TIT.2012.2192713

80. Fehr, S., Berens, S.: On the conditional Rényi entropy. IEEE Trans. Inf. Theory 60(11), 6801 (2014). doi:10.1109/TIT.2014.2357799

81. Tomamichel, M., Berta, M., Hayashi, M.: Relating different quantum generalizations of the conditional Rényi entropy. J. Math. Phys. 55(8), 082206 (2014). doi:10.1063/1.4892761

82. Gigena, N., Rossignoli, R.: Generalized conditional entropy in bipartite quantum systems. J. Phys. A 47(1), 015302 (2014). doi:10.1088/1751-8113/47/1/015302

83. Ollivier, H., Zurek, W.H.: Quantum discord: a measure of the quantumness of correlations. Phys. Rev. Lett. 88(1), 017901 (2001). doi:10.1103/PhysRevLett.88.017901

84. Jurkowski, J.: Quantum discord derived from Tsallis entropy. Int. J. Quantum Inf. 11(01), 1350013 (2013). doi:10.1142/S0219749913500135

85. Bellomo, G., Plastino, A., Majtey, A.P., Plastino, A.R.: Comment on "Quantum discord through the generalized entropy in bipartite quantum states”. Eur. Phys. J. D 68(337), 1 (2014)

86. Berta, M., Seshadreesan, K.P., Wilde, M.M.: Rényi generalizations of quantum information measures. Phys. Rev. A 91(2), 022333 (2015). doi:10.1103/PhysRevA.91.022333 